\title{
An RF Receiver for Intra-Band Carrier Aggregation
}

\author{
Sy-Chyuan Hwu and Behzad Razavi, Fellow, IEEE
}

\begin{abstract}
Carrier aggregation is an attractive approach to increasing the data rate in wireless communication. This paper describes an efficient carrier aggregation receiver architecture that employs one receive path and a single synthesizer. The block-downconversion scalable receiver translates all of the channels to the baseband and utilizes a new digital image rejection technique to reconstruct the signals. A receiver prototype realized in $45 \mathrm{~nm}$ CMOS technology along with an FPGA back end provides an image rejection ratio of at least $70 \mathrm{~dB}$ with a noise figure of $3.8 \mathrm{~dB}$ while consuming $15 \mathrm{~mW}$.
\end{abstract}

Index Terms - Block downconversion, broadband LNA, carrier aggregation, image rejection, LTE.

\section{INTRODUCTION}

I $\mathrm{N}$ ORDER to increase the data rate in wireless communication, two or more adjacent or non-adjacent RF channels can be "joined" together, thus proportionately raising the bandwidth and the capacity. Called "carrier aggregation" [1], this approach has been adopted by the long-term evolution (LTE) standard for cellular systems [2] and poses new RF design challenges. Specifically, the key question is whether for $N$ carriers, one must employ $N$ receivers, transmitters, frequency synthesizers, and baseband chains. It is therefore desirable to develop architectures that can reduce this multiplicity.

This paper proposes a "scalable" receiver architecture based on "block downconversion" and digital image rejection that can support two or more RF carriers using a single receive chain. A CMOS receiver along with an FPGA realization of the background image rejection calibration technique demonstrates a noise figure of $3.8 \mathrm{~dB}$ with a gain of $37 \mathrm{~dB}$ and an image rejection ratio (IRR) of at least $70 \mathrm{~dB}$.

Section II provides the background for this work, presenting the LTE receiver specifications and the prior art. Section III describes the proposed architecture and Section IV the new image rejection algorithm. Sections V and VI deal with the receiver design and experimental results, respectively.

\section{BACKGROUND}

Beyond exploiting bandwidth-efficient modulation schemes, the capacity of wireless links can be raised only by increasing the bandwidth. With the channelization predefined by each standard, this increase can be achieved through decomposing the

\footnotetext{
Manuscript received September 04, 2014; revised November 12, 2014; accepted December 22, 2014. Date of publication February 02, 2015; date of current version March 24, 2015. This paper was approved by Guest Editor Jeffrey Gealow.

The authors are with the Electrical Engineering Department, University of California, Los Angeles, CA 90095-1594 USA (e-mail: razavi@ee.ucla.edu).

Color versions of one or more of the figures in this paper are available online at http://ieeexplore.ieee.org.

Digital Object Identifier 10.1109/JSSC.2014.2386895
}

data that is to be transmitted into two or more streams and impressing them on two or more carriers corresponding to different channels. The RF channels may belong to the same band ("intra-band aggregation") [Fig. 1(a)] or different bands ("interband aggregation") [Fig. 1(b)] [2]. In the former case, the channels can be adjacent to one another or not ("contiguous" and "non-contiguous" aggregation, respectively). In this paper, we consider intra-band aggregation and begin with two channels to illustrate various issues.

\section{A. LTE Specifications}

The LTE receiver requirements are different in the absence or presence of carrier aggregation. In this section, we describe the latter and, specifically, for intra-band aggregation.

For non-contiguous aggregation, LTE specifies a channel bandwidth of $5,10,15,20 \mathrm{MHz}$ with "reference" sensitivities of $-96.5,-93.5,-91.7,-90.5 \mathrm{dBm}$ for QPSK modulation, respectively, and an adjacent channel power $25.5 \mathrm{~dB}$ higher than that of the desired channel [Fig. 2(a)]..$^{1}$ (In the case of contiguous aggregation, the minimum channel bandwidth is $10 \mathrm{MHz}$.) For other in-band blockers, the signal power is set to $12 \mathrm{~dB}$ above the reference sensitivity while the blocker has a power of $-56 \mathrm{dBm}$ if it is at $7.5 \mathrm{MHz}$ offset or $-44 \mathrm{dBm}$ if at $12.5 \mathrm{MHz}$ offset. ${ }^{2}$ Fig. 2(b) and (c) depict examples of contiguous and non-contiguous aggregation for different frequency offsets and different desired channel bandwidths. LTE also stipulates a "narrowband" blocker test wherein an interferer at $-55 \mathrm{dBm}$ is applied at an offset of $0.2 \mathrm{MHz}$, and the desired signal is at a power level of $16 \mathrm{~dB}+$ reference sensitivity [Fig. 2(d)]. The maximum separation between the centers of two intra-band channels is $65 \mathrm{MHz}$.

To maximize spectral usage, LTE allows different bandwidths for the two channels [2] if part of the spectrum is already occupied by another user [Fig. 1(c)]. At the receiver input, that user's signal can be much stronger than the desired channels, thereby acting as a blocker or as an image. In addition, owing to frequency-dependent fading, the two desired channels may arrive with unequal power levels, exhibiting a difference of up to $10 \mathrm{~dB}$ for a $65 \mathrm{MHz}$ separation at $2 \mathrm{GHz}$ [3].

\section{B. Prior Art}

It is possible to dedicate one receiver and one synthesizer to each channel but with a direct power and area penalty. Moreover, with such a small relative frequency separation, the synthesizers must avoid injection pulling, thus dictating a complex

\footnotetext{
${ }^{1}$ These sensitivity levels correspond to band 25 and are specified for intraband non-contiguous carrier aggregation.

${ }^{2}$ Frequency offsets are with respect to the edge of the desired channel.
} 


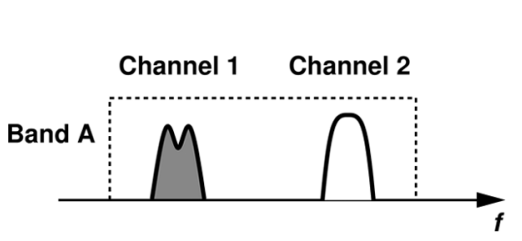

(a)

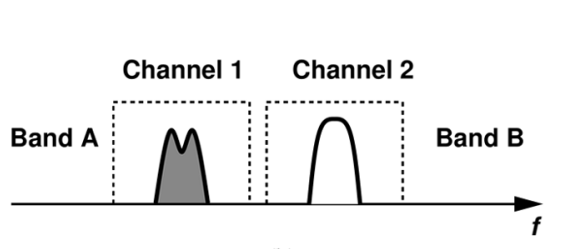

(b)

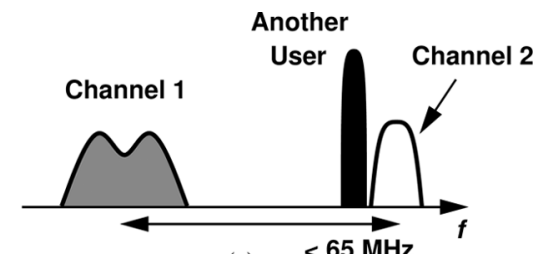

(c)

Fig. 1. (a) Intra-band carrier aggregation, (b) inter-band carrier aggregation, and (c) carrier aggregation in the presence of another user.

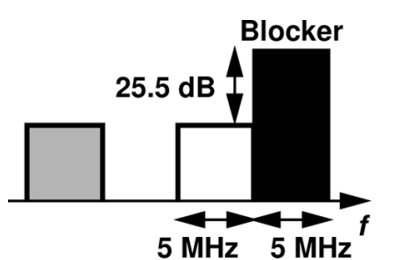

(a)

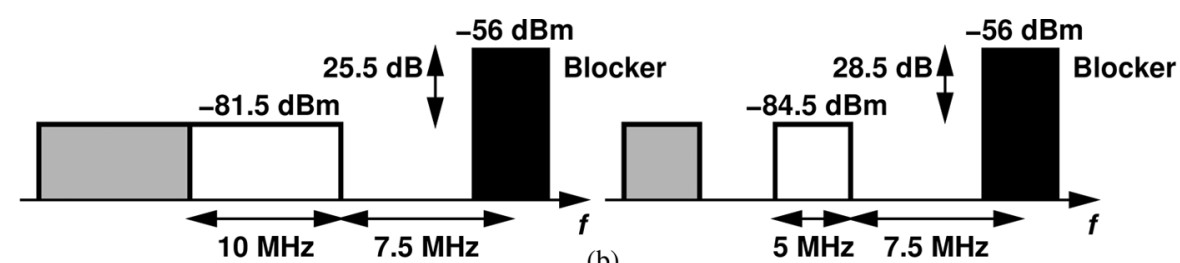

(b)
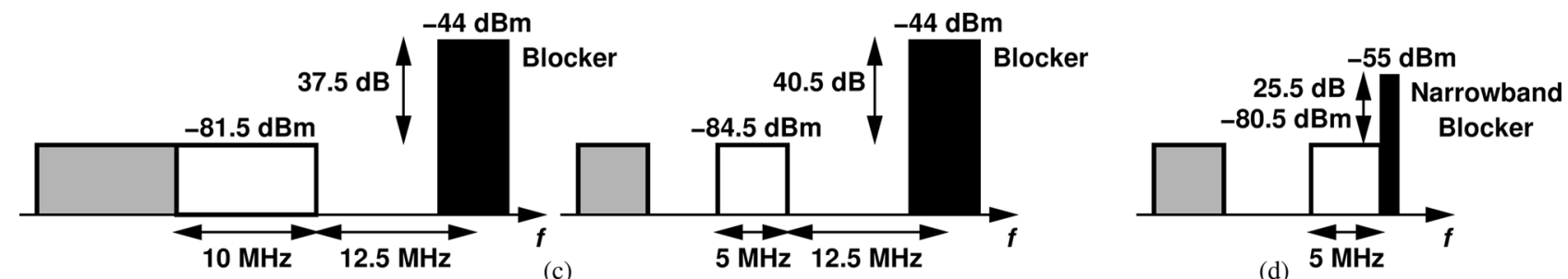

(d) $5 \mathrm{MHz}$

Fig. 2. LTE blocker profiles: (a) adjacent channel, (b) in-band blocking case 1, (c) in-band blocking case 2, and (d) narrowband blocking.

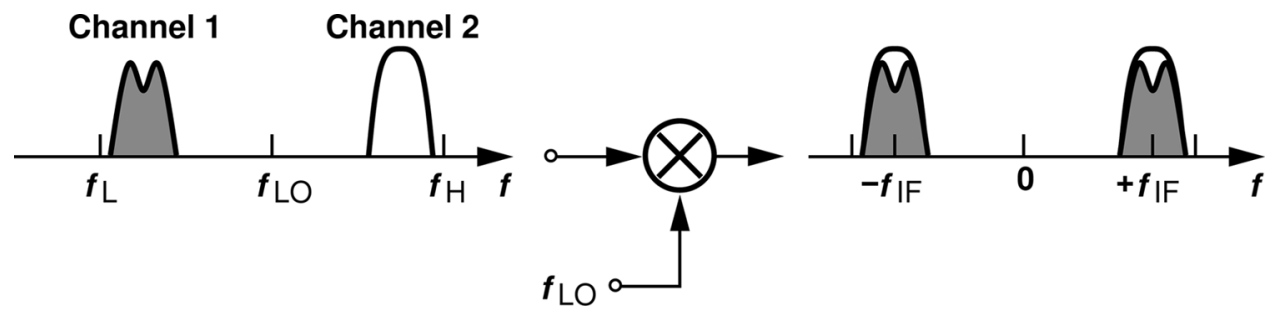

Fig. 3. Block downconversion with two aggregated channels.

frequency plan. For example, to obtain quadrature phases by division, one can operate at twice the channel-1 frequency and the other at four times the channel-2 frequency, but subharmonic or superhamonic injection pulling may still occur.

A more attractive approach is to perform "block downconversion" by a single receiver: the local oscillator (LO) frequency is placed midway between the two channels (Fig. 3), downconverting the entire spectrum from $f_{L}$ to $f_{H}$ and, inevitably, making channel 1 and channel 2 images of each other [4]. While avoiding the power and area penalty of dedicated paths, this method must provide a high image-rejection ratio because, as illustrated in Fig. 1(c), another user's strong signal may act as part of the image and fold onto channel 1 or 2 after downconversion. The image problem is addressed in [4]-[7] in the analog domain. Depicted in simplified form in Fig. 4, this (Weaver) architecture requires a second PLL as well as gain and phase adjustments within the harmonic-reject mixers so as to achieve a high IRR. We shall refer to the first and second downconversion mixers as RF and IF mixers, respectively.

The architecture of Fig. 4 entails three drawbacks. First, it cannot readily guarantee a high IRR across the maximum channel bandwidth (20 MHz in LTE). Second, the IF mixers in Fig. 4 must also suppress blockers that may coincide with the harmonics of the second LO. The design in [4] employs harmonic-reject IF mixers for this purpose.

The third drawback relates to the "scalability" of the architecture, i.e., the growth in power and area as more channels are aggregated. We observe in Fig. 4 that the image-reject/harmonic-reject mixers, the second LO, and the baseband filters and analog-to-digital converters (ADCs) must be duplicated for each additional channel. ${ }^{3}$

\section{Proposed Receiver Architecture}

\section{A. Architecture}

The block downconversion approach illustrated in Fig. 5 can avoid the foregoing three issues if the image rejection is performed in digital domain. Shown at a high level in Fig. 5, the

\footnotetext{
${ }^{3}$ Multiple-input multiple-output (MIMO) applications require duplication of the analog baseband circuits.
} 


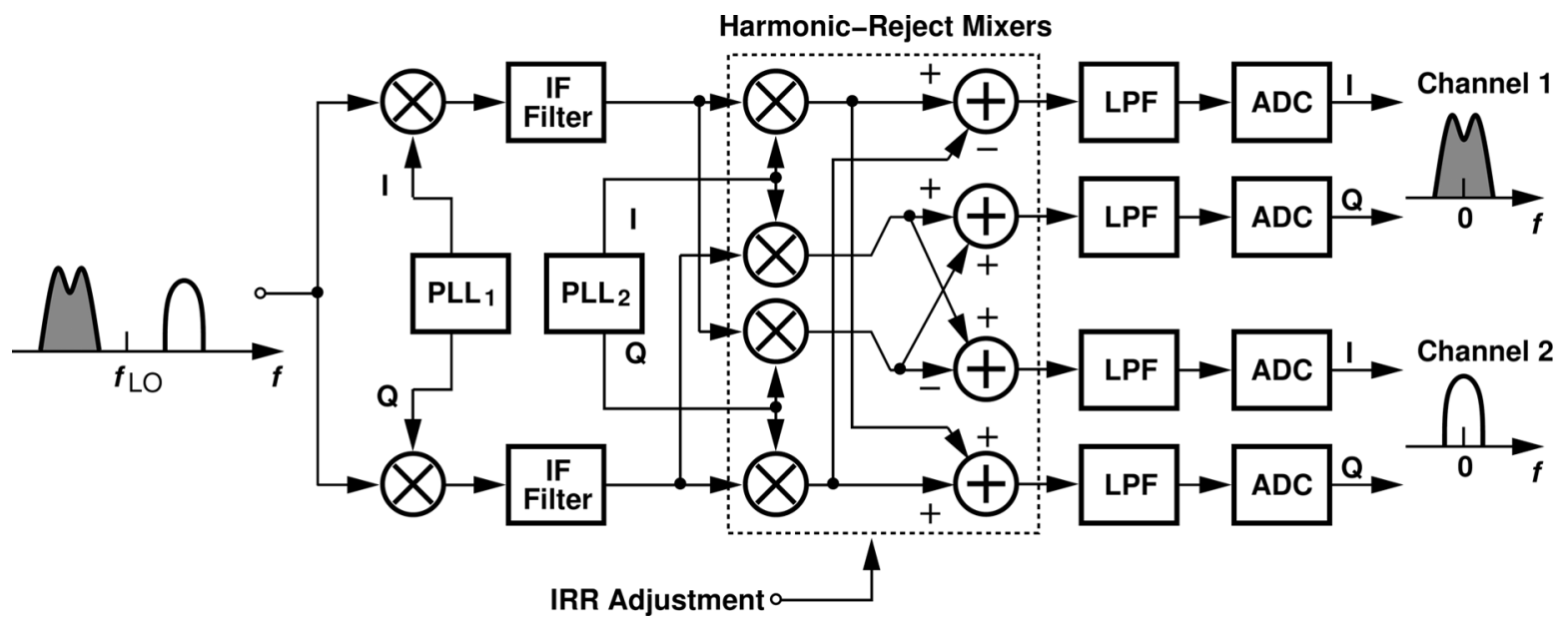

Fig. 4. Receiver in [4] for carrier aggregation.

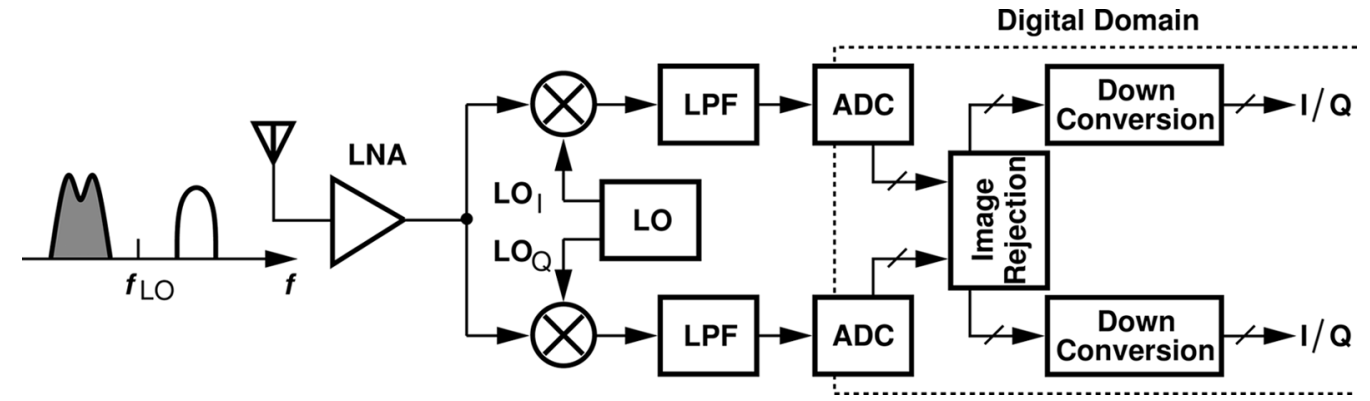

Fig. 5. Proposed block-downconversion receiver for carrier aggregation.

proposed architecture digitizes the quadrature IF signals, removes the image from each channel, and performs downconversion to baseband in the digital domain. The IRR calibration runs in the background (Section IV).

The proposed architecture deals with the three above issues as follows. First, as explained below, the digital image rejection technique inherently accounts for frequency-dependent $\mathrm{I} / \mathrm{Q}$ mismatches. Second, the downconversions in the digital domain incorporate high-purity numerically controlled oscillators (NCOs), in essence multiplying the IF signals by sinusoids ${ }^{4}$ and achieving harmonic-free mixing at much less cost and complexity than analog harmonic-reject mixers. Third, for each channel added to the aggregation, the proposed architecture requires one more digital downconverter and $\mathrm{NCO}$, i.e., an area of about $150 \mu \mathrm{m} \times 150 \mu \mathrm{m}$ and a power consumption of $0.3 \mathrm{~mW}$ in $45 \mathrm{~nm}$ technology (Section V).

\section{B. ADC Requirements}

The proposed architecture incorporates two ADCs to digitize the $35 \mathrm{MHz}$ block of frequencies in the quadrature IF signals. We must then consider the feasibility and power consumption of these ADCs. Among the scenarios depicted in Fig. 2, that containing a $-44 \mathrm{dBm}$ in-band blocker demands the widest ADC dynamic range. (Out-of-band blocking will be discussed later). Let us normalize the voltage gain preceding the ADCs

\footnotetext{
${ }^{4}$ Harmonic-reject mixers also equivalently multiply by an LO waveform that is free from some harmonics.
}

to 1 . For a 64-QAM desired signal at a level of $-84.5 \mathrm{dBm}$ in a $5 \mathrm{MHz}$ bandwidth, the quantization noise of the ADC must remain about $24 \mathrm{~dB}$ below the signal level for a reasonable bit error rate. ${ }^{5}$ Denoting the ADCs' least-significant bit (LSB) size by $\Delta$, we obtain this noise power as $\left[2\left(\Delta^{2} / 12\right) / f_{s}\right] \times 5 \mathrm{MHz}$, where $f_{s}$ is the sampling rate. It follows that

$$
10 \log \left(\frac{2 \Delta^{2}}{12 f_{s}} \times 5 \mathrm{MHz}\right)=-84.5 \mathrm{dBm}-24 \mathrm{~dB}
$$

For example, if $f_{s}=70 \mathrm{MHz}$, then $\Delta=7.7 \mu \mathrm{V}$.

The upper end of the ADCs' dynamic range is dictated by the $-44 \mathrm{dBm}$ blocker level, i.e., a full scale of $4 \mathrm{mV}$. (Of course, both the LSB size and the full scale should be multiplied by the receiver gain.) In addition, the spurs generated by such a blocker must also fall below $-84.5 \mathrm{dBm}-24 \mathrm{~dB} \approx-109 \mathrm{dBm}$. We conclude that the ADCs must provide a resolution of 9 bits and a spurious-free dynamic range (SFDR) of $-44 \mathrm{dBm}-(-109 \mathrm{dBm})=65 \mathrm{~dB}$, i.e., a linearity of about 11 bits. In practice, an additional margin of $10 \mathrm{~dB}$ is necessary to account for the RX front-end noise, peak-to-average power ratio of interferers and other imperfections.

Fortunately, recent advances in high-performance ADC design make our proposed solution a plausible one. For example, [9] reports a 14 bit, $80 \mathrm{MHz}$ converter that achieves an SFDR

\footnotetext{
${ }^{5}$ In [8], the required error vector magnitude for 64-QAM is at least $-22 \mathrm{~dB}$.
} 
of $80 \mathrm{~dB}$ at the Nyquist rate while consuming $31 \mathrm{~mW}$. Such an $\mathrm{ADC}$ offers both a resolution and a dynamic range for exceeding the above results with a modest power consumption.

Operating in a full-duplex system, an LTE receiver must deal with the transmitter leakage, which can be as high as $-20 \mathrm{dBm}$ at $15 \mathrm{MHz}$ below the RX band. This leakage and other outof-band blockers can be attenuated by low-pass filters having a bandwidth of $35 \mathrm{MHz}$ (not included in this work) after downconversion to IF to the point that they are less than about $50 \mathrm{~dB}$ above the desired signal (e.g., around $-30 \mathrm{dBm}$ ).

With a moderate attenuation of the out-of-band blockers, the ADC must cope with aliasing. To this end, one can utilize the oversampling ADC in [10], which achieves an SFDR of $77 \mathrm{~dB}$ at $37.5 \mathrm{MHz}$ while drawing $39 \mathrm{~mW}$.

\section{Proposed Image Rejection Algorithm}

In the case of the LO frequency placed midway between the two aggregated carriers, the worst-case image rejection corresponds to the narrowband blocker scenario shown in Fig. 2(d) and a 64-QAM desired signal. The blocker must be rejected by at least $-55 \mathrm{dBm}-(-80.5 \mathrm{dBm}-24 \mathrm{~dB}) \approx 50 \mathrm{~dB}$. Nevertheless, when the number of wanted signals exceeds 2 , the required IRR should be obtained from Fig. 2(c), resulting in $-44 \mathrm{dBm}-(-84.5 \mathrm{dBm}-24 \mathrm{~dB}) \approx 65 \mathrm{~dB}$.

A number of self-calibrating image rejection techniques have been reported [11]-[15]. Among these, [12] achieves the highest IRR, $62 \mathrm{~dB}$, but it does not calibrate frequency-dependent I/Q mismatches, a critical issue if the signal and the image can appear anywhere in a wide bandwidth (from nearly zero to $35 \mathrm{MHz}$ in our case).

\section{A. Frequency-Dependent I/Q Mismatch}

The I and Q signal paths following the first downconversion in Fig. 5 include various filtering sections. To appreciate the effect of frequency-dependent mismatches, let us consider a first-order low-pass RC network as a representative circuit. If the resistor and capacitor mismatches between the I and Q paths produce a small pole frequency mismatch of $\Delta \omega_{0}$, then the corresponding transfer functions can be written as $H_{I}(s)=\left(1+s / \omega_{0}\right)^{-1}$ and $H_{Q}(s)=\left[1+s /\left(\omega_{0}+\Delta \omega_{0}\right)\right]^{-1}$, yielding a gain mismatch of $\epsilon=$ $\left|H_{Q}\right| /\left|H_{I}\right|-1 \approx\left(\Delta \omega_{0} / \omega_{0}\right) \omega^{2} /\left(\omega^{2}+\omega_{0}^{2}\right)$ and a phase mismatch of $\theta=\tan ^{-1}\left(\omega / \omega_{0}\right)-\tan ^{-1}\left[\omega /\left(\omega_{0}+\Delta \omega_{0}\right)\right] \approx \omega \Delta \omega_{0} /\left(\omega^{2}+\right.$ $\left.\omega_{0}^{2}\right)$. It follows that IRR ${ }^{-1} \approx\left(\epsilon^{2}+\theta^{2}\right) / 4 \approx(1 / 4)\left[\omega^{2} /\left(\omega^{2}+\right.\right.$ $\left.\left.\omega_{0}^{2}\right)\right]\left(\Delta \omega_{0} / \omega_{0}\right)^{2}$. As an example, Fig. 6 plots $10 \log ($ IRR $)$ across the band for $\Delta \omega_{0} / \omega_{0}=2 \%$, displaying considerable deterioration as the frequency exceeds one-tenth of the pole frequency. We therefore observe the extremely tight matching necessary for maintaining an IRR of greater than, say, $60 \mathrm{~dB}$ across the band.

It is possible to employ multi-tap adaptive filters to calibrate the mismatches in the background [15], but the analysis in [16] proves the existence of a "phantom" solution and the resulting convergence issues. For this reason, [15] limits the number of taps to two, affording frequency response correction at only two points.

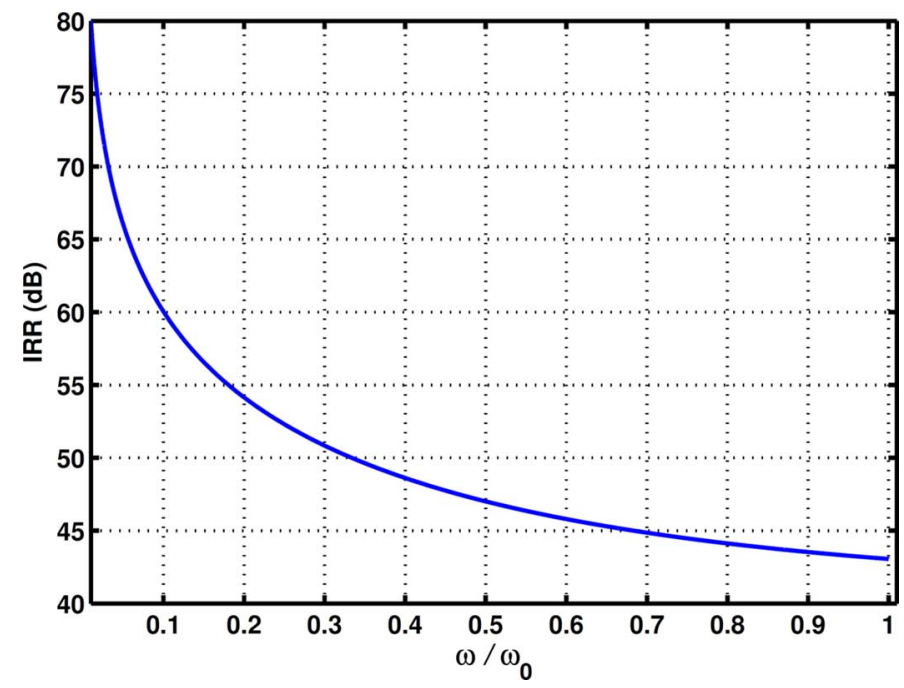

Fig. 6. Frequency-dependent image rejection ratio for $\Delta \omega_{0} / \omega_{0}=2 \%$.

\section{B. Proposed Algorithm}

Consider the perfectly balanced downconverter shown in Fig. 7(a), where two channels A and B are received and translated to one IF. ${ }^{6}$ In order to visualize the image components, we examine the complex signal, $I(\omega)-j Q(\omega)$, which carries $\mathrm{A}$ and $\mathrm{B}$ in negative and positive frequencies, respectively. With perfect matching, A and B are free from each other's images. To produce the baseband signals, the I and Q signals can be downconverted again using an LO frequency equal to $\omega_{I F}$ and properly combined. For example, the in-phase baseband component of $\mathrm{A}, x_{A, I}(t)$, is obtained by forming $I(t) \cos \omega_{\mathrm{IF}} t+Q(t) \sin \omega_{\mathrm{IF}} t$ and low-pass filtering the result.

In the presence of gain and phase mismatches, as modeled in Fig. 7(b) by $\epsilon$ and $\theta$, respectively, the composite signal $I(\omega)-$ $j Q(\omega)$ exhibits a finite corruption of A by B and vice versa. This corruption is given by a coefficient equal to $\epsilon \exp (j \theta)$.

Let us now make a key observation: two of the spectral components of $Q(\omega)$ in Fig. 7(b) are multiplied by $\alpha=(1+$ $\epsilon) \exp (j \theta)$. We thus surmise that, if the spectral components of $I(\omega)$ are also subjected to the same scaling factor, then the images may be removed. As illustrated in Fig. 7(c), we multiply $I(\omega)$ by $(1+\epsilon) \exp (j \theta)$ in the frequency domain, ${ }^{7}$ obtaining a signal that upon combining with $\mathrm{Q}$, cancels the images.

The proposed method can simplify the problem of image rejection, and, more generally, I/Q calibration. However, we must first develop a means of measuring $\alpha$ in the background. An important point in our proposition is that $\epsilon$ and $\theta$ need not be measured individually for $\alpha$ to be computed. Let us assume that the digitized I and Q signals are first applied to FFT engines, providing $I(\omega)$ and $Q(\omega)$ as the only observable quantities. We wish to obtain $\alpha$ in terms of $I(\omega)$ and $Q(\omega)$. If $P_{A}$ and $P_{B}$ respectively denote the total power of the $\mathrm{A}$ and $\mathrm{B}$ channels in Fig. 7(c), we recognize that the average power of $|I(\omega)|$ is in fact

\footnotetext{
${ }^{6} \mathrm{We}$ use the notations $\mathrm{A}$ and $\mathrm{B}$ to refer to both the RF channels and the IF channels.

${ }^{7}$ Frequency-domain multiplication is denoted by a cross in a square to avoid confusion with time-domain multiplication.
} 


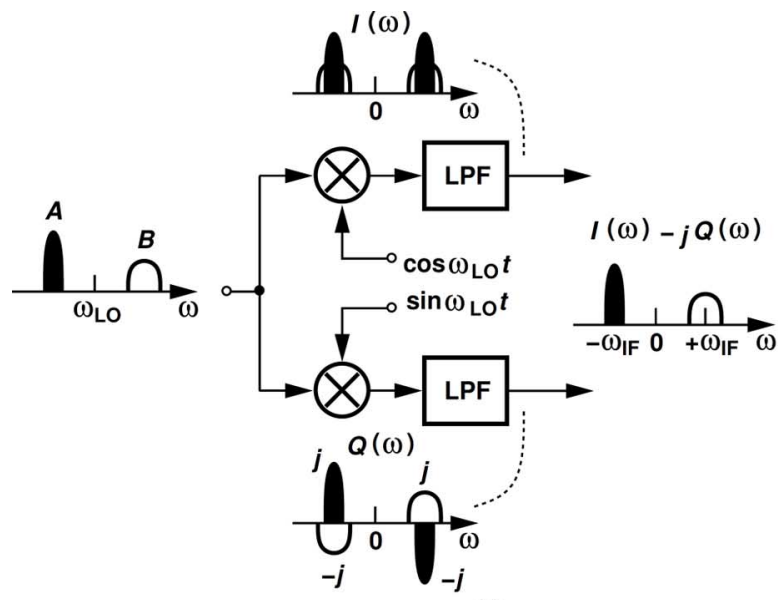

(a)

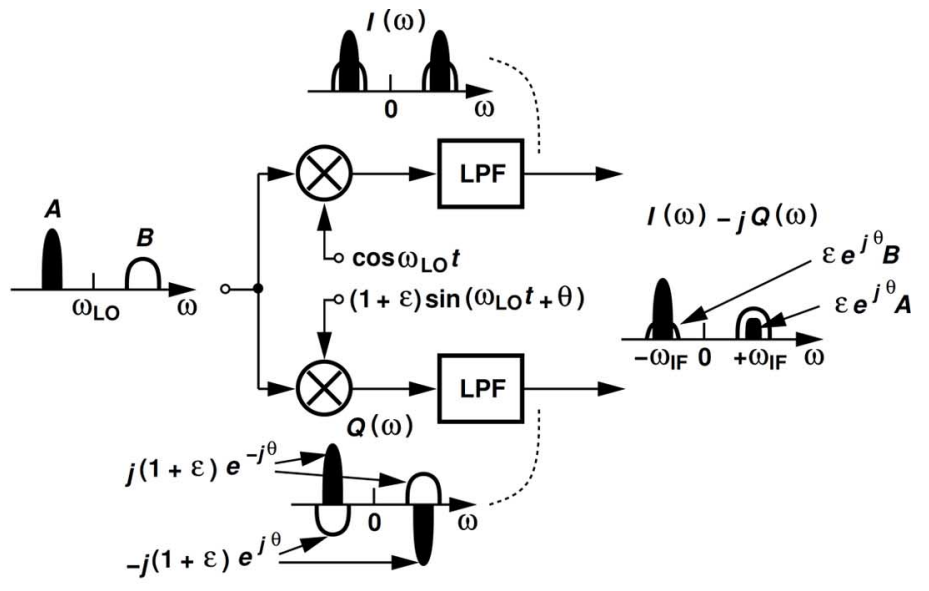

(b)

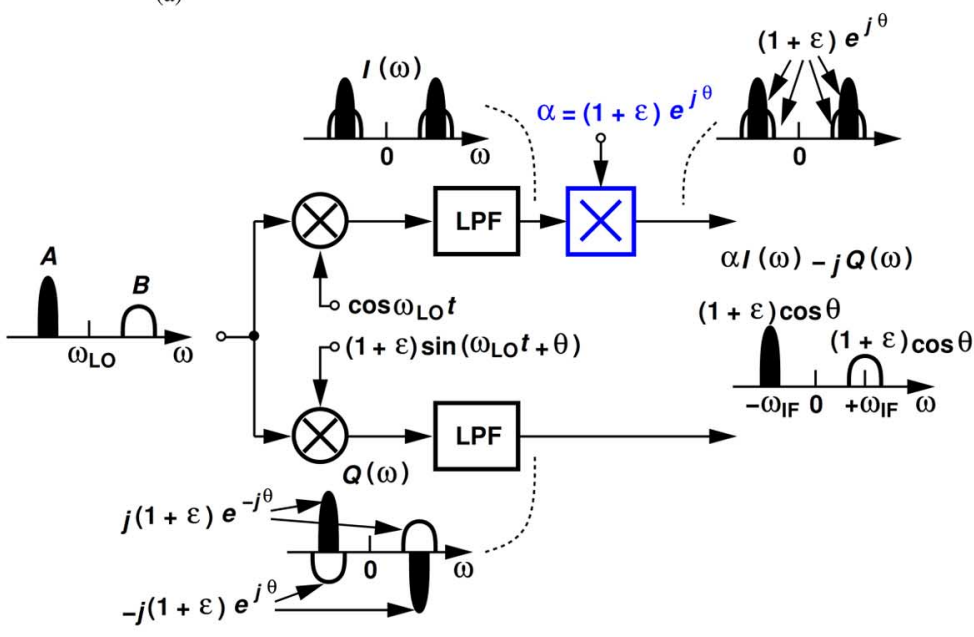

(c)

Fig. 7. (a) Ideal image-reject receiver, (b) image problem due to I/Q mismatch, and (c) proposed image rejection technique.

equal to $E\left\{|I(\omega)|^{2}\right\}=P_{A}+P_{B}$, where $E\{\cdot\}$ is the expectation value, the gain from the antenna to the ADC output is normalized to unity, and $\mathrm{A}$ and $\mathrm{B}$ are assumed statistically independent. Similarly, $E\left\{|Q(\omega)|^{2}\right\}=(1+\epsilon)^{2}\left(P_{A}+P_{B}\right)$. In addition, it can be shown that the average value of the inner product of $I(\omega)$ and $Q(\omega)$ is given by $E\{I(\omega) \cdot Q(\omega)\}=(1+\epsilon)\left(P_{A}+P_{B}\right) \sin \theta$. We thus express $\alpha=(1+\epsilon) \cos \theta+j(1+\epsilon) \sin \theta$ as

$$
\begin{aligned}
& \operatorname{Im}\{\alpha\}=\frac{E\{I(\omega) \cdot Q(\omega)\}}{E\left\{|I(\omega)|^{2}\right\}} \\
& \operatorname{Re}\{\alpha\}=\sqrt{\frac{E\left\{|Q(\omega)|^{2}\right\}}{E\left\{|I(\omega)|^{2}\right\}}-\left[\frac{E\{I(\omega) \cdot Q(\omega)\}}{E\left\{|I(\omega)|^{2}\right\}}\right]^{2}} .
\end{aligned}
$$

While appearing computationally formidable, (2) and (3) allow us to calculate $\alpha$ so long as the I and Q signals are not zero.

Fig. 8 summarizes the necessary digital operations at a high level. In order to reproduce the corrected $I(t)$, an inverse FFT (IFFT) engine follows the complex scaling operation. If the functions prescribed by (2) and (3) can be realized at an acceptable cost, then background image rejection (or I/Q calibration) is achieved entirely in the digital domain. Remarkably, since $\operatorname{Im}\{\alpha\}$ and $\operatorname{Re}\{\alpha\}$ can be calculated for each FFT bin, the frequency-dependent mismatches are corrected with a fine frequency resolution. We also observe that the complexity in Fig. 8 is independent of the number of aggregated channels.

The accuracy with which $\alpha$ is calculated trades with the time over which $\operatorname{Im}\{\alpha\}$ and $\operatorname{Re}\{\alpha\}$ are averaged. In this work, the averaging time is about $1 \mathrm{~ms}$ to ensure a minimum IRR of $70 \mathrm{~dB}$.

\section{RECEIVER DESIGN}

Fig. 9 shows the experimental receiver designed and implemented in this work as a demonstration vehicle. The receiver consists of 1) a CMOS prototype realizing the RF front-end and baseband amplification, 2) off-the-shelf low-pass filters and ADCs, and 3) an FPGA implementation of the image rejection algorithm and IF downconverters. The ADCs have a nominal resolution of 14 bits and a maximum sampling rate of $250 \mathrm{MHz}$ [17] (but run at $50 \mathrm{MHz}$ here due to the setup constraints). The $\mathrm{RF}$ front-end receives an external LO signal at twice the desired carrier frequency and generates the $25 \%$ LO phases that drive the mixers.

Shown in Fig. 10, the front-end consists of a broadband LNA, passive mixers, and transimpedance amplifiers (TIAs). The sections below describe the analysis and design of the receiver building blocks. 


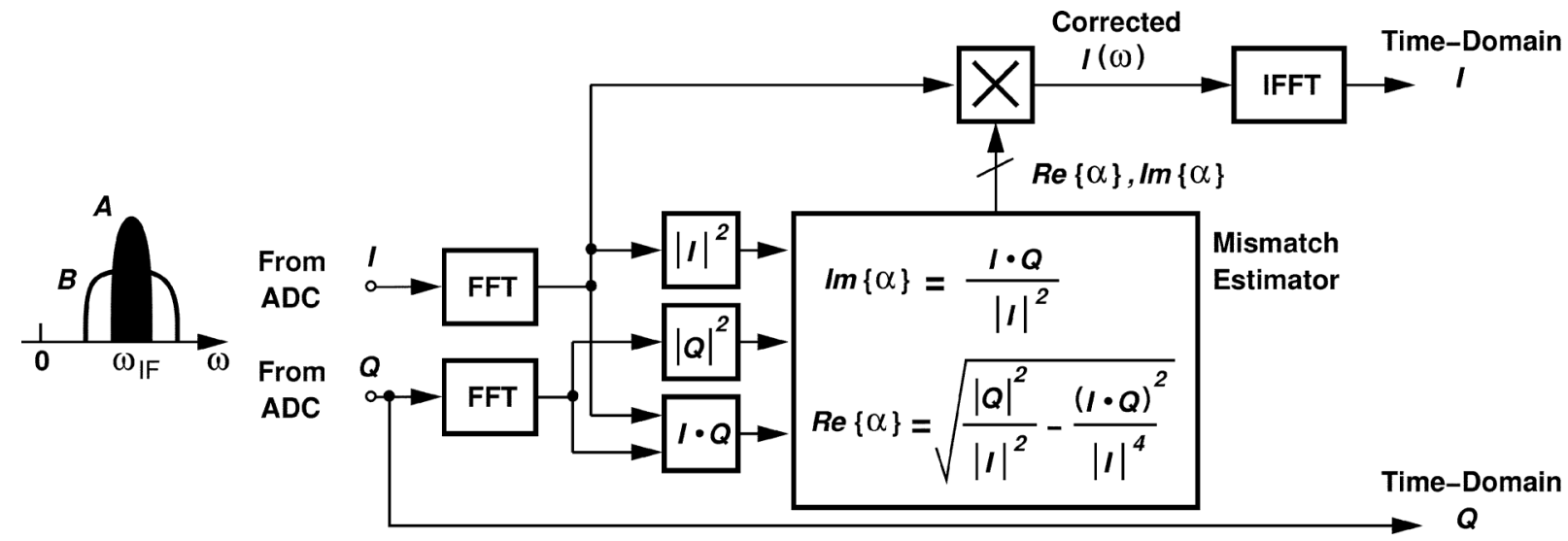

Fig. 8. Proposed image-reject algorithm.

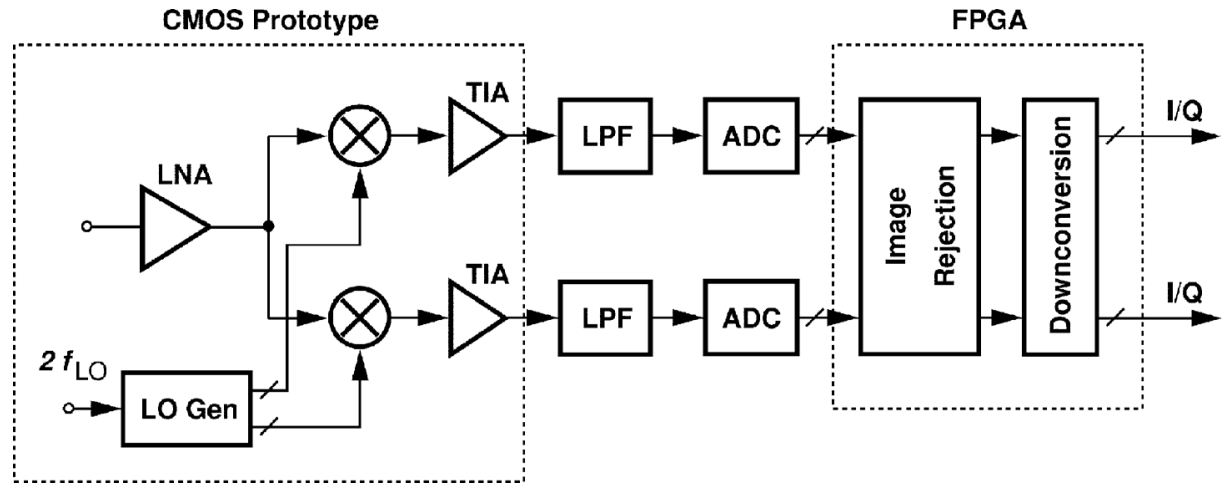

Fig. 9. Experimental receiver implementation.

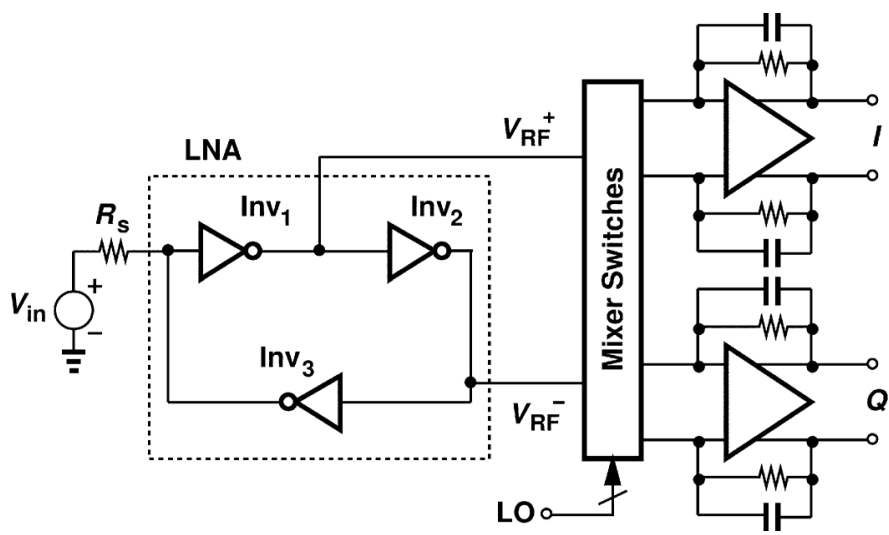

Fig. 10. Proposed RF front-end circuit.

\section{A. Proposed Low-Noise Amplifier}

The use of CMOS inverters as LNAs goes back to the early 1990s [18] and has also been practiced in a number of other topologies [19], especially as a $G_{m}$ stage preceding current-driven mixers. We seek an inductor-less LNA that performs single-ended to differential conversion, provides input matching, and operates with a low supply. It has been suggested that a multi-stage LNA with feedback exhibits input matching with a wide bandwidth [20]. We therefore propose the topology shown in Fig. 11(a), where active feedback by means of Inv 3 affords a low noise figure and broadband matching. In this circuit, $R_{\mathrm{mix}}$ denotes the input resistance of the downconversion mixers and is chosen low $(\approx 50 \Omega)$ so as to minimize the LNA internal voltage swings, thereby improving its linearity in a manner similar to other topologies [19]. The RF signals at nodes $\mathrm{X}$ and $\mathrm{Y}$ are approximately differential.

With the low value of $R_{\text {mix }}$, the boundary between the LNA and the mixers begins to diminish as most of the RF currents produced by $\operatorname{Inv}_{1}$ and $\operatorname{Inv}_{2}$ are absorbed by the mixers. Nonetheless, we first analyze the stand-alone LNA and then explore the properties of the LNA/mixer cascade. The analysis and design of the circuit begin with enforcing input matching and the hope that the resulting voltage gain and noise figure will be acceptable.

For the input resistance to be equal to $R_{S}$, we arrive at the following condition $G_{m 1} R_{\text {mix }} G_{m 2} R_{\text {mix }} G_{m 3} R_{S}=1$, where channel-length modulation is neglected. We then obtain the closed-loop voltage gains to nodes $\mathrm{X}$ and $\mathrm{Y}$ as $\left|V_{X} / V_{\text {in }}\right|=$ $G_{m 1} R_{\text {mix }} / 2$ and $\left|V_{Y} / V_{\text {in }}\right|=G_{m 1} R_{\text {mix }} G_{m 2} R_{\text {mix }} / 2$. It follows that the currents delivered to the mixers at $\mathrm{X}$ and $\mathrm{Y}$ are equal to $\left(G_{m 1} / 2\right) V_{\text {in }}$ and $\left(G_{m 1} G_{m 2} R_{\text {mix }} / 2\right) V_{\text {in }}$, respectively. For $V_{X}$ and $V_{Y}$ to be differential, $G_{m 2} R_{\text {mix }}=1$.

\section{B. LNA Biasing}

While offering flexibility in the design, the LNA's three inverters experience considerable PVT-induced variation in their bias current. It is possible to define the bias current by placing 


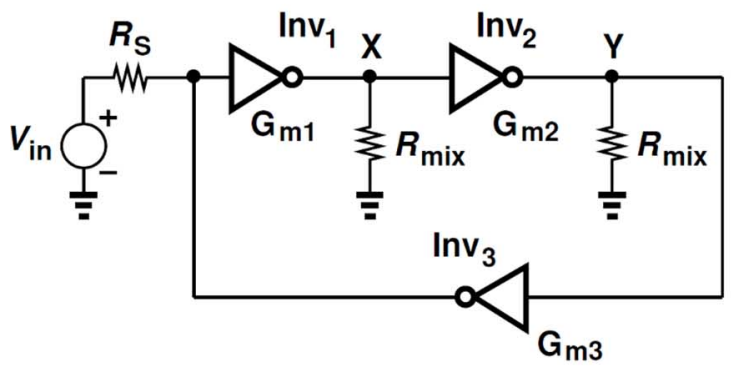

(a)

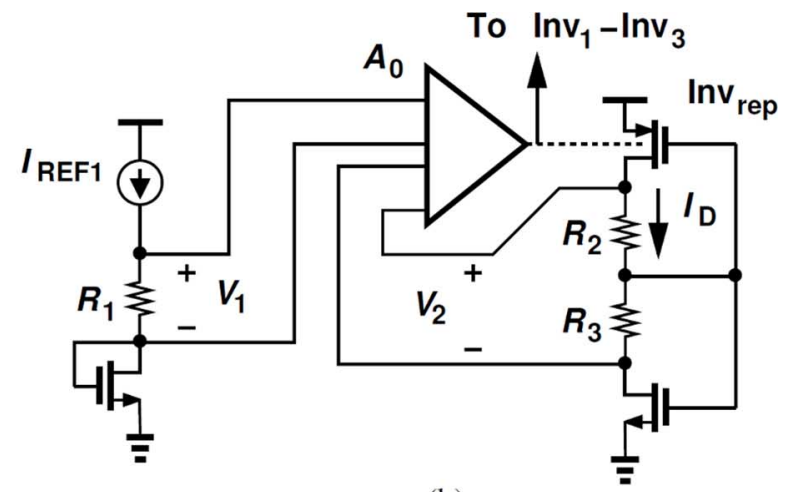

(b)

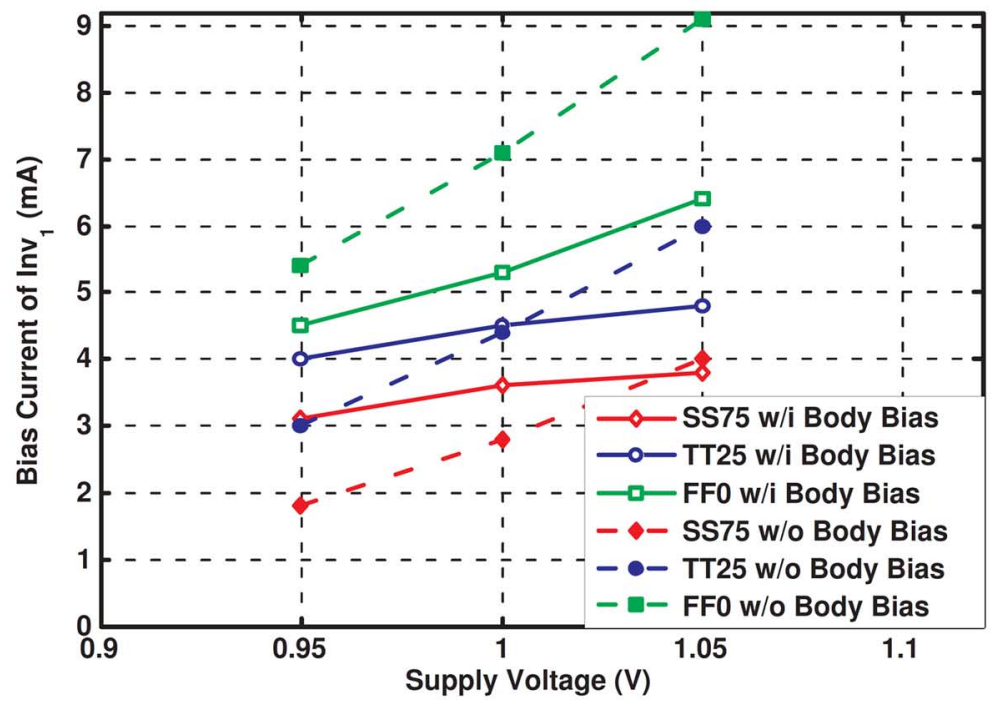

(c)

Fig. 11. (a) Proposed LNA topology, (b) PMOS body bias circuit, and (c) bias current of Inv 1 with and without PMOS body bias.

a current source in series with the PMOS or NMOS devices [21] but at the cost of voltage headroom and hence linearity. We propose a method of controlling the bias that does not sacrifice headroom.

Illustrated in Fig. 11(b), the idea is to adjust the bias current of a replica inverter, $\operatorname{Inv}_{\text {rep }}$, through the PMOS body voltage. To achieve a well-defined value for $I_{D}$, a servo loop consisting of $A_{0}$ forces the voltage $V_{2}=\left(R_{2}+R_{3}\right) I_{D}$ to be equal to $V_{1}=R_{1} I_{\mathrm{REF} 1}$ and hence $I_{D}=\left[R_{1} /\left(R_{2}+R_{3}\right)\right] I_{\mathrm{REF} 1}$. The output voltage of $A_{0}$ drives the PMOS bodies of $\operatorname{Inv}_{1}-\operatorname{Inv}_{3}$, faithfully copying $I_{D}$ onto the three stages if mismatches are acceptably small.

Due to channel-length modulation, $V_{2}$ must be small enough to avoid a large mismatch between $\operatorname{Inv}_{\text {rep }}$ and $\operatorname{Inv}_{1}-\operatorname{Inv}_{3}$. On the other hand, $V_{2}$ must be sufficiently larger than the input offset of $A_{0}$. In this work, $V_{2}=150 \mathrm{mV}$ as a compromise and $A_{0}=$ 16.5. Fig. 11(c) plots the simulated bias current of $\operatorname{Inv}_{1}$ as a function of $V_{D D}$ for several process and temperature corners with and without the servo loop, revealing a fourfold reduction provided by the proposed technique. Although the bias current still varies considerably, the input matching, voltage gain, and noise figure are relatively constant because the input impedance of the mixers also varies but in a favorable direction.

\section{TIA Design}

The design of the TIA is influenced by the LNA and mixers. Since random mismatches in Fig. 10 may create a difference between the dc levels of $V_{\mathrm{RF}}^{+}$and $V_{\mathrm{RF}}^{-}$, the mixer switches carry a dc current, thereby producing substantial flicker noise. With the low impedance seen at the input of the mixers, capacitive coupling proves difficult, especially at the lower edge of the LTE band $(700 \mathrm{MHz})$. This issue is resolved through the use of two TIAs as shown in Fig. 12(a) so as to isolate $V_{\mathrm{RF}}^{+}$and $V_{\mathrm{RF}}^{-}$. In this case, the mismatch between the TIAs' common-mode level and the dc level of $V_{\mathrm{RF}}^{+}$or $V_{\mathrm{RF}}^{-}$does produce a dc current through the switches, but this current is limited by the TIA's feedback resistor. For example, a $50 \mathrm{mV}$ dc difference generates $10 \mu \mathrm{A}$ and hence negligible flicker noise.

To avoid doubling the bias current as a result of decomposing the TIA into two, we implement the two using different MOSFET types and share their bias current, as shown in Fig. 12(b). Due to limited voltage headroom, the PMOS pair's bias is defined through its body rather than by a tail current source.

The required third-order intercept point $\left(\operatorname{IIP}_{3}\right)$ can be derived from Fig. 13. According to [2], the desired signal at $-84.5 \mathrm{dBm}$ 


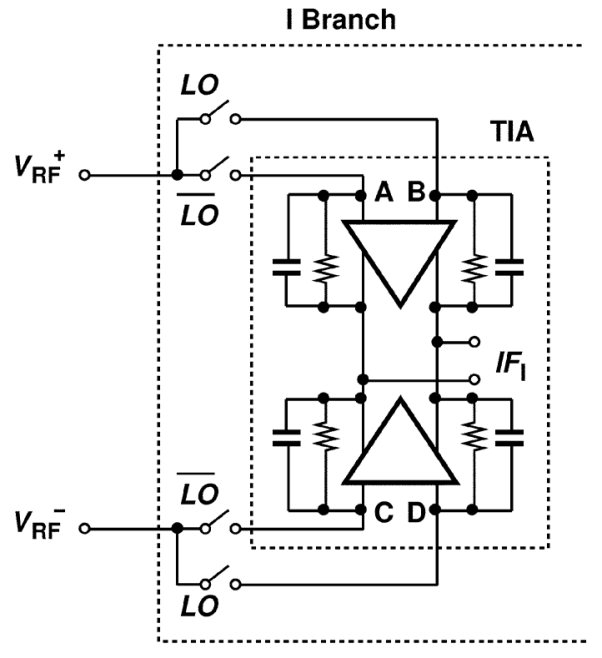

(a)

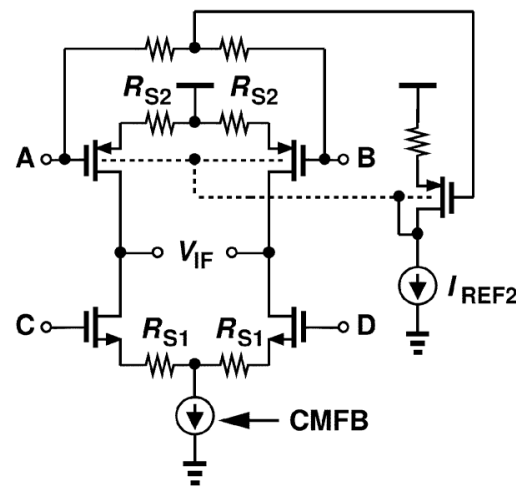

(b)

Fig. 12. (a) Separate TIAs to avoid DC current and (b) amplifier implementation.

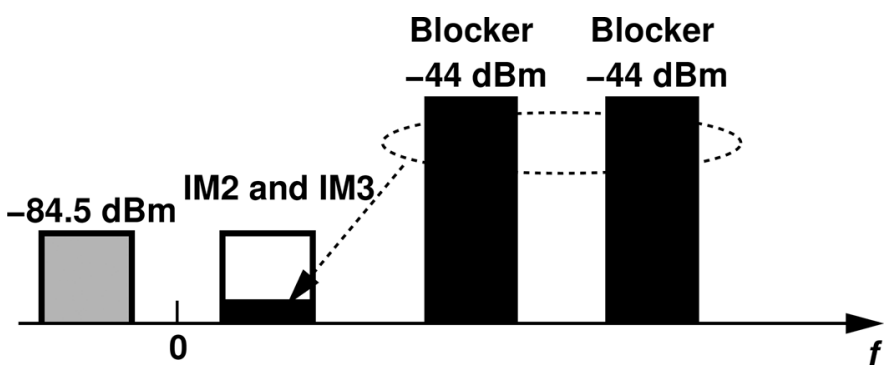

Fig. 13. Intermodulation in the baseband.

could be accompanied by a $-44 \mathrm{dBm}$ in-band blocker. The intermodulation term should be below the desired signal by about $24 \mathrm{~dB}$ for 64-QAM modulation. We conclude that the required receiver IIP 3 should exceed $-12 \mathrm{dBm}$. The degeneration resistors, $R_{S 1}=30 \Omega$ and $R_{S 2}=60 \Omega$, in Fig. 12(b) and a bias current of $2 \mathrm{~mA}$ establish the requisite linearity.

\section{Single-Ended Noise Analysis}

In this and the next sections, we analyze the noise performance of the receiver and demonstrate an important property of the LNA. The analysis proceeds in three steps: (1) downconvert the broadband LNA noise to the IF, (2) add the TIA noise to the result, and (3) refer the overall RX output noise to the LNA input and determine the NF. While the method in [24] is also applicable here, our approach more easily allows the inclusion of the baseband stages following the TIA. As explained in the previous section, we assume $G_{m 2} R_{\text {mix }}=1$ and $G_{m 1} G_{m 2} G_{m 3} R_{\mathrm{mix}}^{2} R_{S}=1$, i.e., $G_{m 3} R_{S}=\left(G_{m 1} R_{\mathrm{mix}}\right)^{-1}$.

Fig. 14 shows the LNA circuit and the noise components, $I_{n 1}-I_{n 3}$, contributed by $\operatorname{Inv}_{1}-\mathrm{Inv}_{3}$, respectively. We assume the TIA is single-ended and connected to $\mathrm{X}$. Since the impedance seen at $\mathrm{X}$ is equal to $R_{\text {mix }} /\left(1+G_{m 1} G_{m 2} G_{m 3} R_{\text {mix }}^{2} R_{S}\right)=R_{\text {mix }} / 2$, the noise voltage at this node due to $I_{n 1}$ is equal to $I_{n 1} R_{\text {mix }} / 2$. We also multiply the noise voltage at $\mathrm{P}, I_{n 3} R_{S} / 2$, by $G_{m 1} R_{\text {mix }}$ and

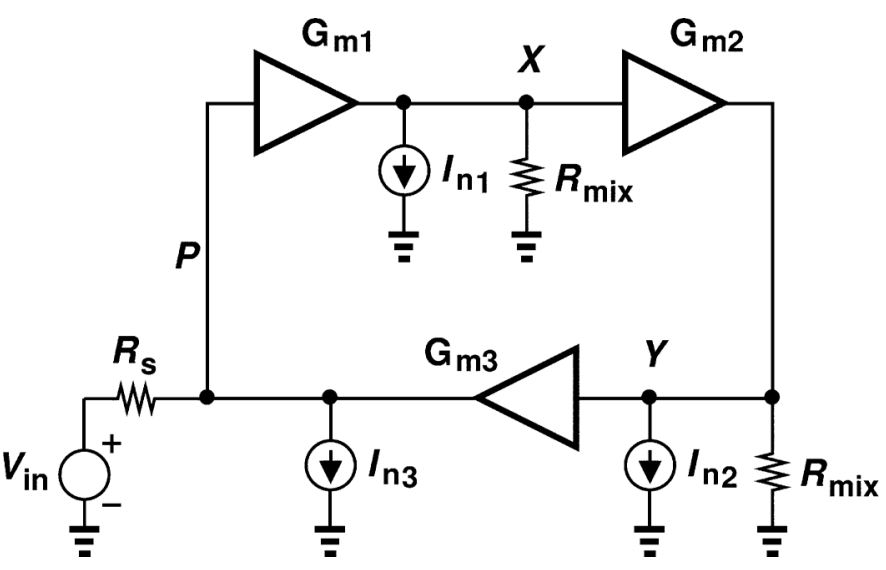

Fig. 14. LNA noise model.

that at $\mathrm{Y}$, by $G_{m 3} R_{S} R_{m 1} R_{\mathrm{mix}}$, obtaining the noise voltage at $\mathrm{X}$ due to $I_{n 3}$ and $I_{n 2}$. The total noise at this node thus equals

$$
\overline{V_{n, X}^{2}}=\frac{R_{\operatorname{mix}}^{2}}{4}\left[\overline{I_{n 1}^{2}}+\overline{I_{n 2}^{2}}+\left(G_{m 1} R_{S}\right)^{2} \overline{I_{n 3}^{2}}\right] .
$$

To downconvert the above RF noise to IF, we construct the model shown in Fig. 15(a), where $R_{\mathrm{LNA}}$ denotes the LNA output impedance and $R_{\mathrm{SW}}$ the switch on-resistance. The IF devices $R_{\text {TIA }}$ and $C_{1}$ represent the single-ended input impedance of the TIA. Since $R_{\mathrm{LNA}} \approx R_{\text {mix }}$, the noise voltage source is modeled as $2 V_{n, X}$, yielding $V_{n, X}$ at node $\mathrm{M}$ (which would be the same as node $X$ in Fig. 14). In this design, $R_{\mathrm{TIA}} C_{1}(=25 \mathrm{~ns})$ is much greater than the LO period, $T_{\mathrm{LO}}$, so as to maximize the receiver voltage gain.

The principal difficulty facing our noise analysis is that the broadband LNA produces noise at the harmonics of the LO, and the input impedance of the downconversion mixers varies substantially at these harmonics. In this design, both $R_{\mathrm{TIA}} C_{1} \gg$ $T_{\mathrm{LO}}$ and $\left(R_{\mathrm{LNA}}+R_{\mathrm{SW}}\right) C_{1} \gg T_{\mathrm{LO}}$, leading to current-driven 


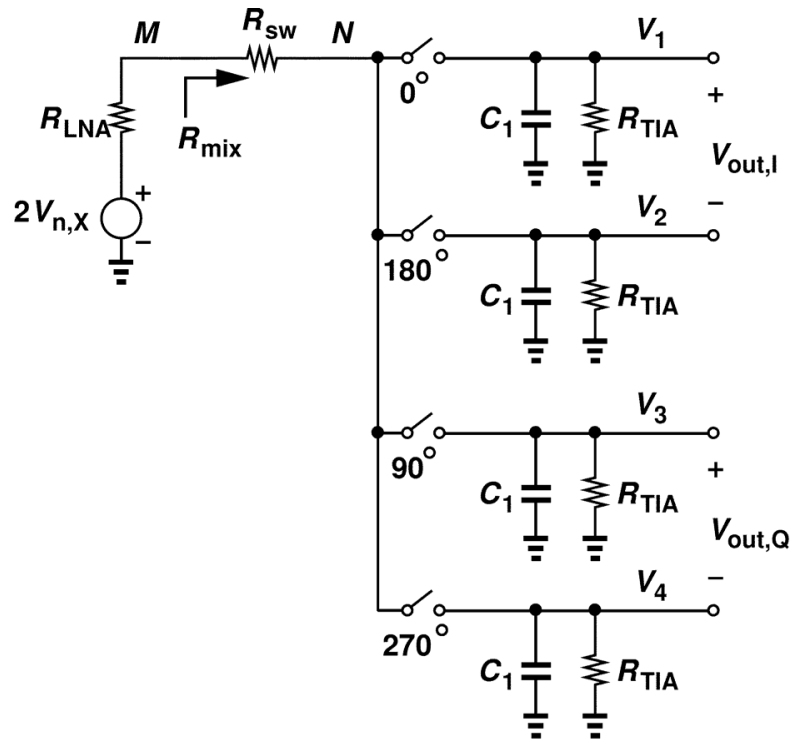

(a)

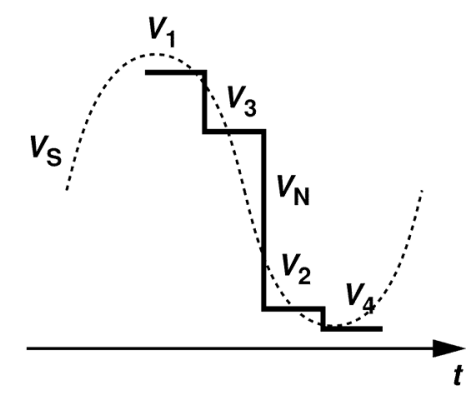

(b)

Fig. 15. Downconversion noise model.

mixing. To determine the conversion gain at different harmonic frequencies, let us replace the noise source in Fig. 15(a) with $V_{S}=V_{0} \cos \left(N \omega_{\mathrm{LO}} t\right)$ and examine the waveform at node $\mathrm{N}$. Due to the condition $\left(R_{\mathrm{LNA}}+R_{\mathrm{SW}}\right) C_{1} \gg T_{\mathrm{LO}}$, the output voltages vary by a small amount when each switch turns on. We equate the charge lost by $C_{1}$ through $R_{\mathrm{TIA}}$ in the hold mode to the charge delivered by $V_{S}$ to $C_{1}$ in the tracking mode [24]:

$$
\frac{V_{k} T_{\mathrm{LO}}}{R_{\mathrm{TIA}}}=\int_{t_{1}}^{t_{2}} \frac{V_{S}-V_{k}}{R_{\mathrm{LNA}}+R_{\mathrm{SW}}} d t(k=1, \ldots, 4),
$$

where $t_{2}-t_{1}=T_{\mathrm{LO}} / 4$. It can be proved that the amplitude of the differential IF outputs in Fig. 15(a) is given by

$$
\begin{aligned}
\left|V_{1}-V_{2}\right| & =\left|V_{3}-V_{4}\right| \\
& =\frac{1}{N} \frac{4 \sqrt{2}}{\pi} \frac{R_{\mathrm{TIA}} V_{0}}{R_{\mathrm{TIA}}+4 R_{\mathrm{LNA}}+4 R_{\mathrm{SW}}} \operatorname{odd} N, \\
& =0 \text { even } N .
\end{aligned}
$$

This result suggests that the downconversion to differential outputs has the same $1 / N$ gain dependence as simple return-to-zero passive 50\%-duty-cycle mixers but with a different coefficient. The broadband noise power at odd $\mathrm{LO}$ harmonics can therefore be multiplied by $1+1 / 3^{2}+1 / 5^{2}+\cdots=\pi^{2} / 8$ and the square of $(4 \sqrt{2} / \pi) R_{\mathrm{TIA}} /\left(R_{\mathrm{TIA}}+4 R_{\mathrm{LNA}}+4 R_{\mathrm{SW}}\right)$.

From the foregoing observations and noting that $R_{\mathrm{LNA}}$ in Fig. 15 is equal to $R_{\text {mix }}$, we can express the downconverted LNA noise appearing in $V_{\text {out }, \mathrm{I}}=V_{1}-V_{2}$ as

$$
\overline{V_{n, I}^{2}}=\frac{4 \pi^{2}}{8}\left(\frac{4 \sqrt{2}}{\pi} \frac{R_{\mathrm{TIA}}}{R_{\mathrm{TIA}}+4 R_{\mathrm{LNA}}+4 R_{\mathrm{SW}}}\right)^{2} \overline{V_{n, X}^{2}},
$$

which, from (4), reduces to

$$
\begin{aligned}
\overline{V_{n, I}^{2}}=4\left(\frac{R_{\mathrm{TIA}}}{R_{\mathrm{TIA}}+4 R_{\mathrm{LNA}}+4 R_{\mathrm{SW}}}\right)^{2} \\
R_{\text {mix }}^{2}\left[\overline{I_{n 1}^{2}}+\overline{I_{n 2}^{2}}+\left(G_{m 1} R_{S}\right)^{2} \overline{I_{n 3}^{2}}\right] .
\end{aligned}
$$

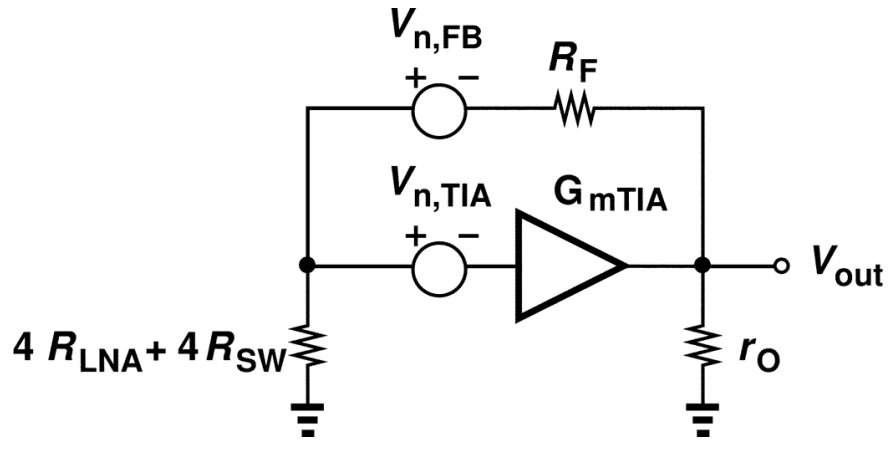

Fig. 16. Model for TIA noise calculation.

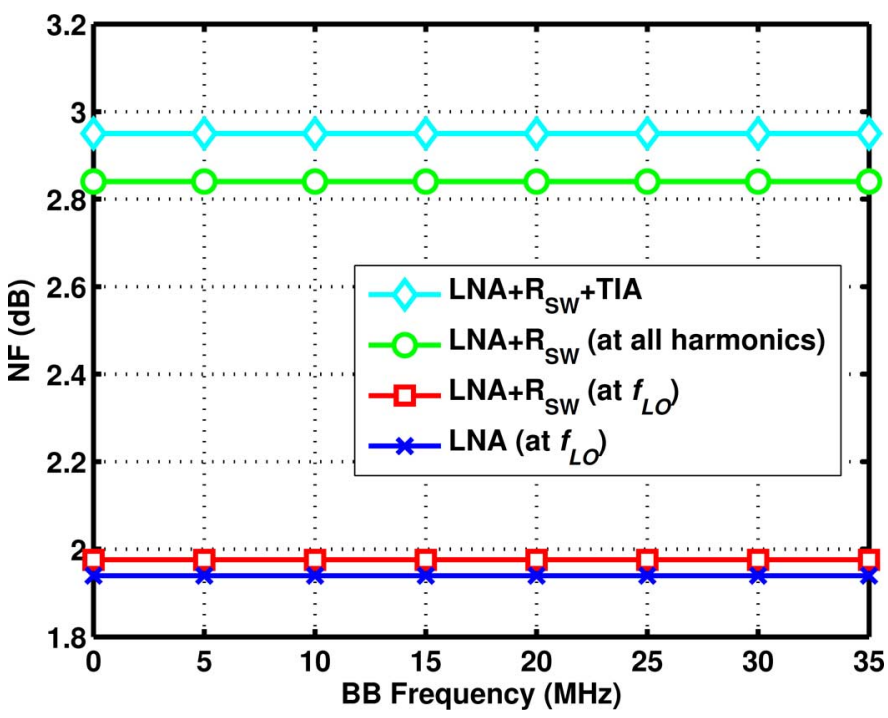

Fig. 17. Noise figure simulation results.

The baseband noise of $R_{\mathrm{SW}}$ is downconverted in the same manner:

$$
\overline{V_{n, \text { out }, S W}^{2}}=4 \overline{V_{n, S W}^{2}}\left(\frac{R_{\mathrm{TIA}}}{R_{\mathrm{TIA}}+4 R_{\mathrm{LNA}}+4 R_{\mathrm{SW}}}\right)^{2},
$$

where $\overline{V_{n, S W}^{2}}=4 k T R_{\mathrm{SW}}$. 


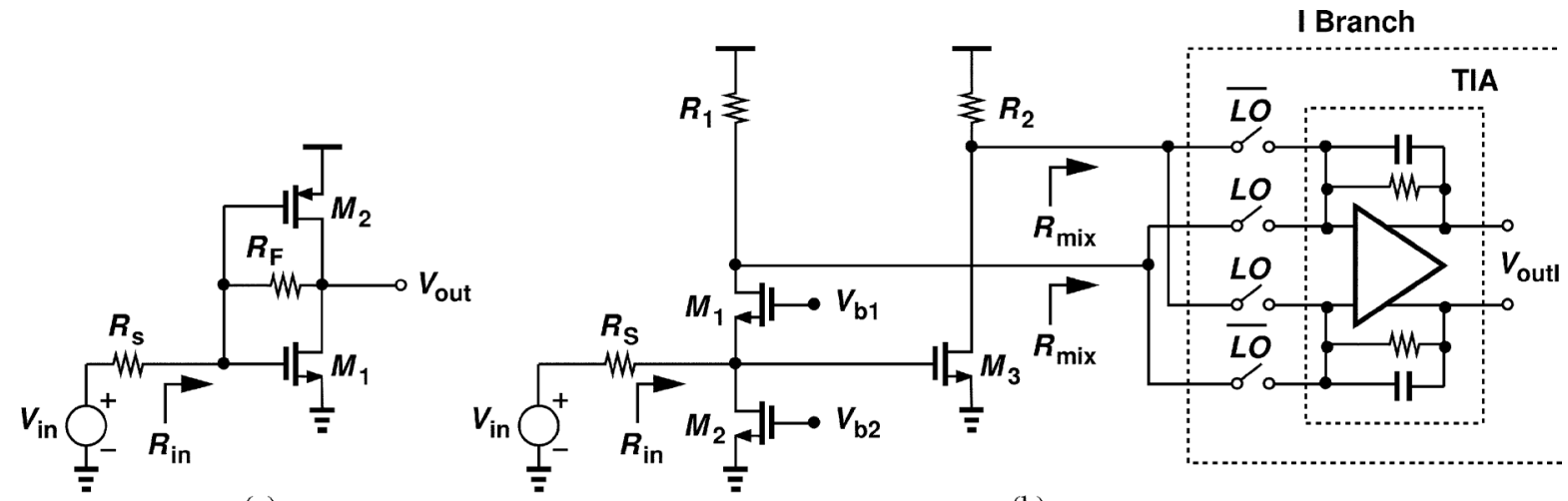

(a)

(b)

Fig. 18. (a) Resistive-feedback LNA and (b) noise-cancelling LNA.

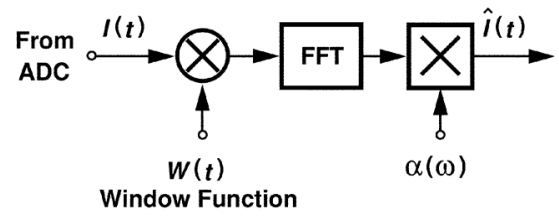

(a)

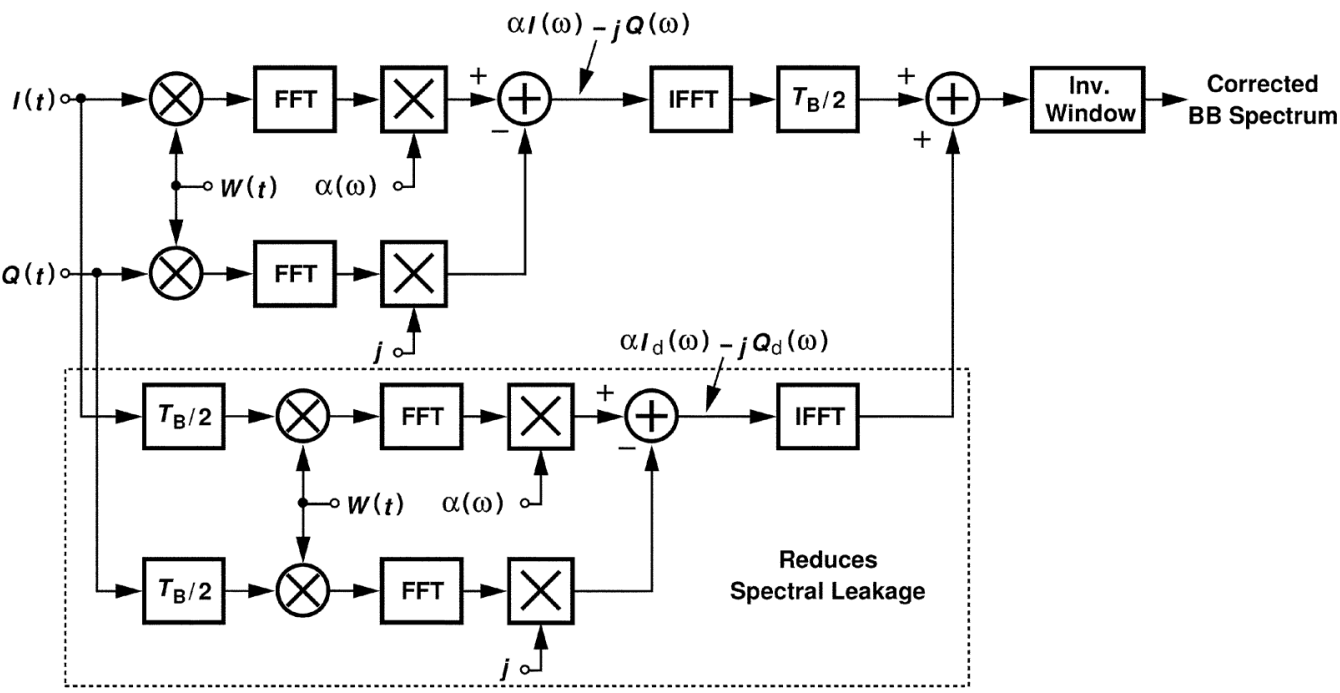

(b)

Fig. 19. (a) Window function multiplication, and (b) shifting path to suppress spectral leakage.

We must now calculate the noise contributed by the TIA. As shown in Fig. 16, with a $25 \%$ duty cycle, each single-ended input of the TIA sees an equivalent driving impedance of $4 R_{\mathrm{LNA}}+4 R_{\mathrm{SW}}$. The output noise arising from $R_{F}$ and the core amplifier can be expressed as

$$
\begin{gathered}
\left.\overline{V_{n, \text { out }}^{2}}\right|_{\mathrm{res}+\mathrm{amp}}=\frac{\left[1+G_{m \mathrm{TIA}}\left(4 R_{\mathrm{LNA}}+4 R_{\mathrm{SW}}\right)\right]^{2}}{D^{2}} \overline{V_{n, F B}^{2}} \\
+\frac{G_{m \mathrm{TIA}}^{2}\left(R_{F}+4 R_{\mathrm{LNA}}+4 R_{\mathrm{SW}}\right)^{2}}{D^{2}} \overline{V_{n, \text { TIA }}^{2}},
\end{gathered}
$$

where $D=1+G_{m \text { TIA }}\left(4 R_{\mathrm{LNA}}+4 R_{\mathrm{SW}}\right)+\left(R_{F}+4 R_{\mathrm{LNA}}+\right.$ $\left.4 R_{\mathrm{SW}}\right) / r_{O}$. The total output noise is equal to the components given by (8) and (9) times $A_{0}^{2}$ plus that given by (10), where $A_{0}$ is the voltage gain from the input of the core amplifier to its output. The voltage gain from the antenna to the TIA output is given by

$$
A_{\mathrm{tot}}=G_{m 1} R_{\mathrm{mix}}\left(\frac{4 \sqrt{2}}{\pi} \frac{R_{\mathrm{TIA}}}{R_{\mathrm{TIA}}+4 R_{\mathrm{mix}}+4 R_{\mathrm{SW}}}\right) A_{\mathrm{TIA}} .
$$

We must now divide the output noise by $4 k T R_{S} A_{\text {tot }}^{2}$ and substitute for $\overline{I_{n 1,2,3}^{2}}$ to find the noise figure. We have in Fig. 14: $\overline{I_{n 1}^{2}}=4 k T \gamma G_{m 1}$ with no contribution from $R_{\text {mix }}$ because 


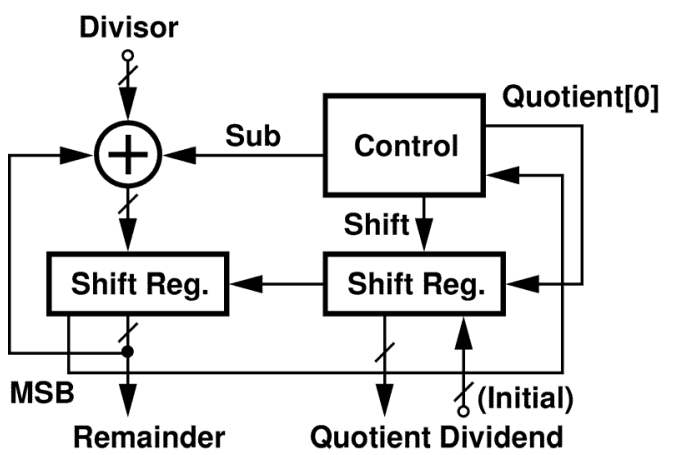

(a)

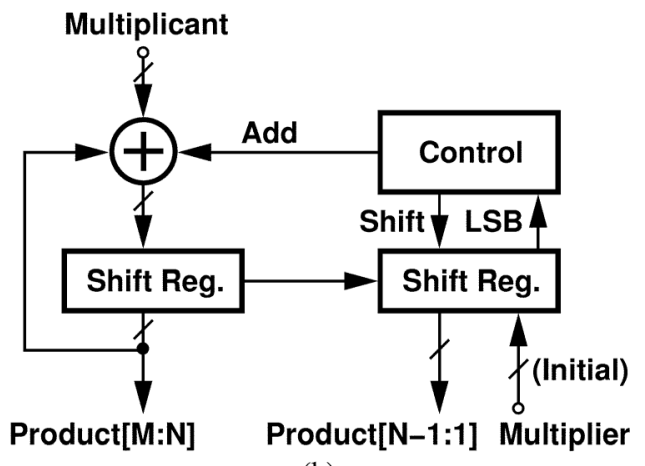

(b)

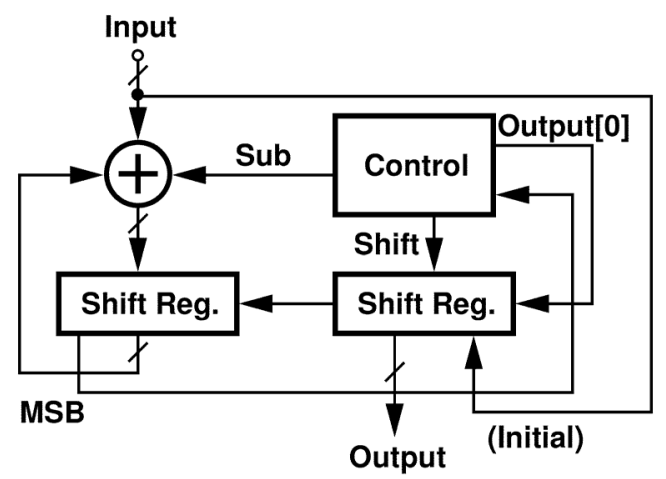

(c)

Fig. 20. Bit-serial arithmetic implementations of (a) division, (b) multiplication, and (c) square root functions.

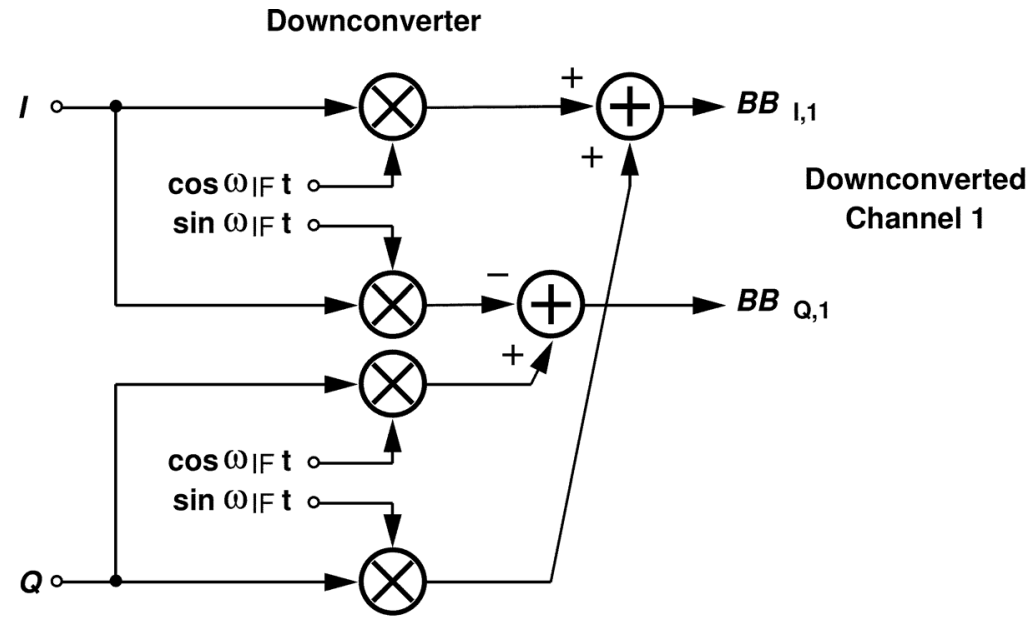

16-Bit Multiplier

(a)

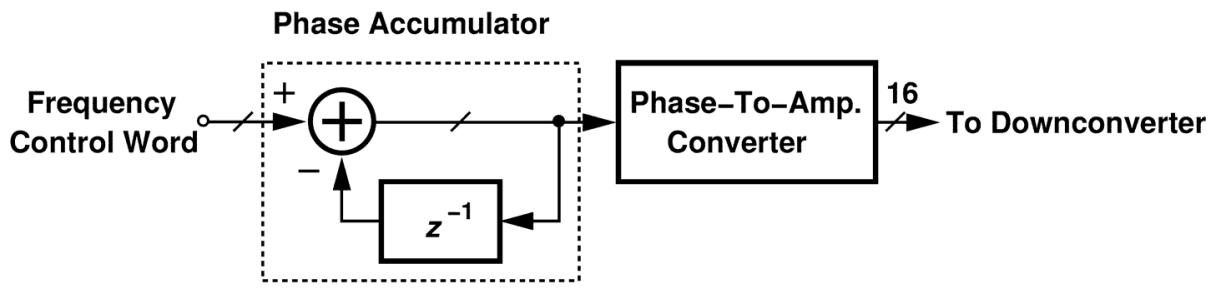

(b)

Fig. 21. (a) Digital downconverter, and (b) numerically-controlled oscillator. 


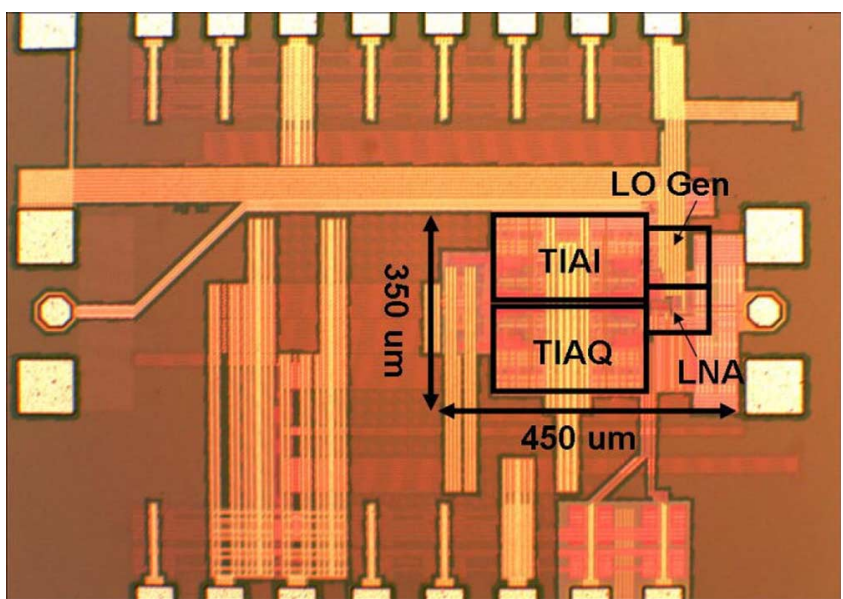

Fig. 22. Front-end die photograph.

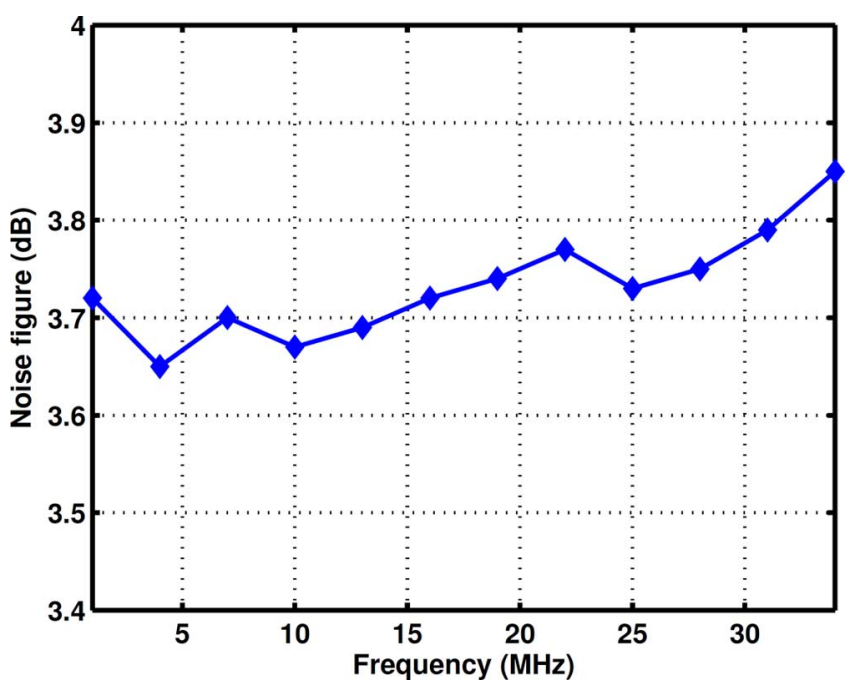

Fig. 23. Measured noise figure.

the switch resistance noise is included above. For $\overline{I_{n 2}^{2}}$, on the other hand, we write $\overline{I_{n 2}^{2}} \approx 4 k T \gamma\left(G_{m 2}+1 / R_{\text {mix }}\right)$, because the switch resistance comprises $80 \%$ of $R_{\text {mix }}$. Finally, we have $\overline{I_{n 3}^{2}}=4 k T \gamma\left(G_{m 3}+1 / R_{S}\right)$. It follows that

$$
\begin{aligned}
& N F= \\
& \frac{\pi^{2}}{8}\left[1+\frac{\gamma}{G_{m 1} R_{S}}+\frac{\gamma+1}{G_{m 1}^{2} R_{S} R_{\mathrm{mix}}}+\frac{\gamma}{G_{m 1} R_{\mathrm{mix}}}+\frac{R_{\mathrm{SW}}}{R_{S}\left(G_{m 1} R_{\mathrm{mix}}\right)^{2}}\right] \\
& +\frac{2 R_{F}}{R_{S}} \frac{\left[1+G_{m \mathrm{TIA}}\left(4 R_{\mathrm{LNA}}+4 R_{\mathrm{SW}}\right)\right]^{2}}{A_{\mathrm{tot}}^{2} D^{2}} \\
& +2 \frac{G_{m \mathrm{TIA}}^{2}\left(R_{F}+4 R_{\mathrm{LNA}}+4 R_{\mathrm{SW}}\right)^{2}}{D^{2}} \frac{\overline{V_{n, \mathrm{TIA}}}}{4 k T R_{S} A_{\mathrm{tot}}^{2}}
\end{aligned}
$$

In order to show the relative contributions of the receiver's devices, Fig. 17 plots the NF for four cases: 1) only the LNA has noise, 2) the LNA and switch resistance have noise at $f_{\mathrm{LO}}, 3$ ) the LNA and switch resistance have noise at all LO harmonics, and

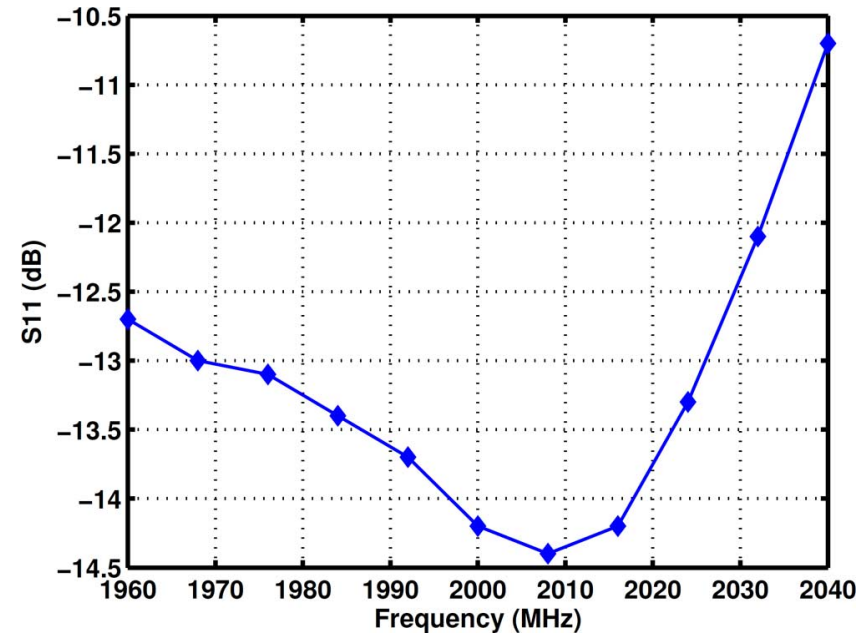

Fig. 24. Measured input matching.

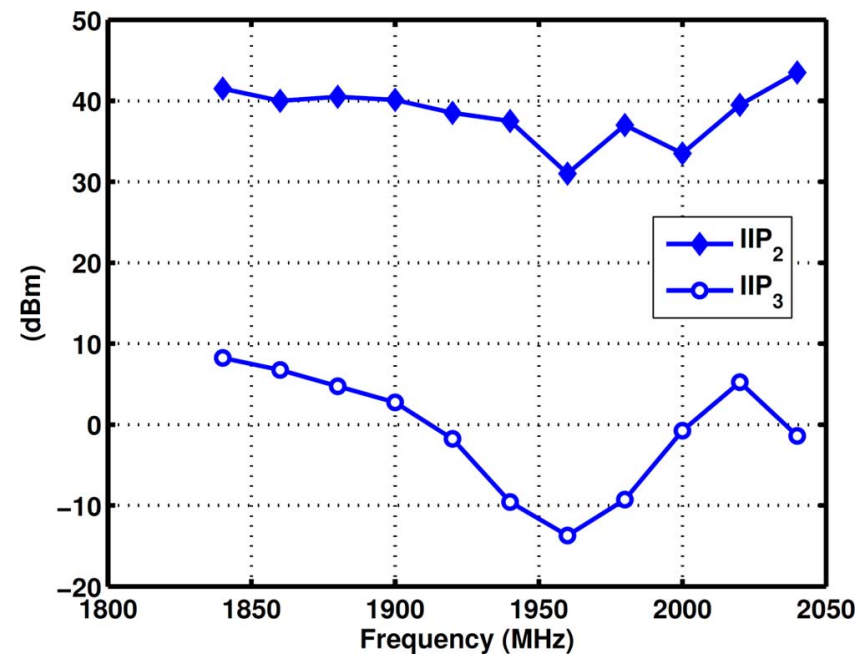

Fig. 25. Measured $I I P_{2}$ and $I I P_{3}$.

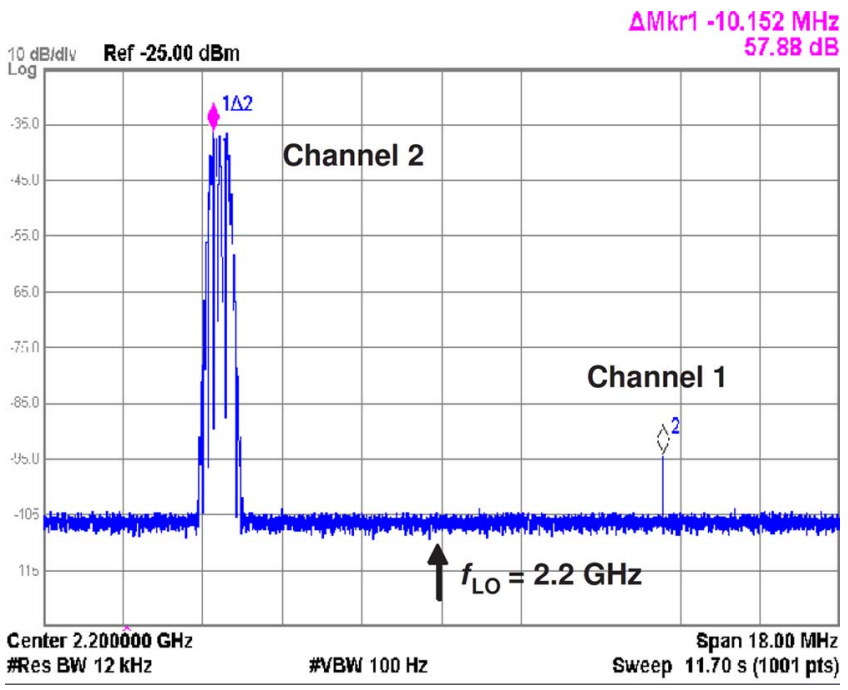

Fig. 26. Measured RF spectrum with two carriers.

4) the entire receiver. It is worth noting that the inclusion of noise at harmonics raises the NF by $0.9 \mathrm{~dB}$. 


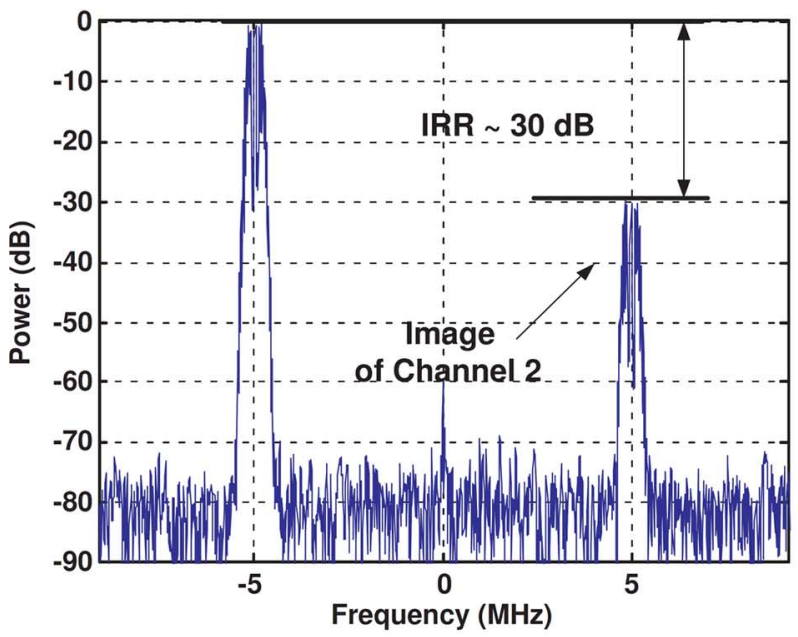

(a)

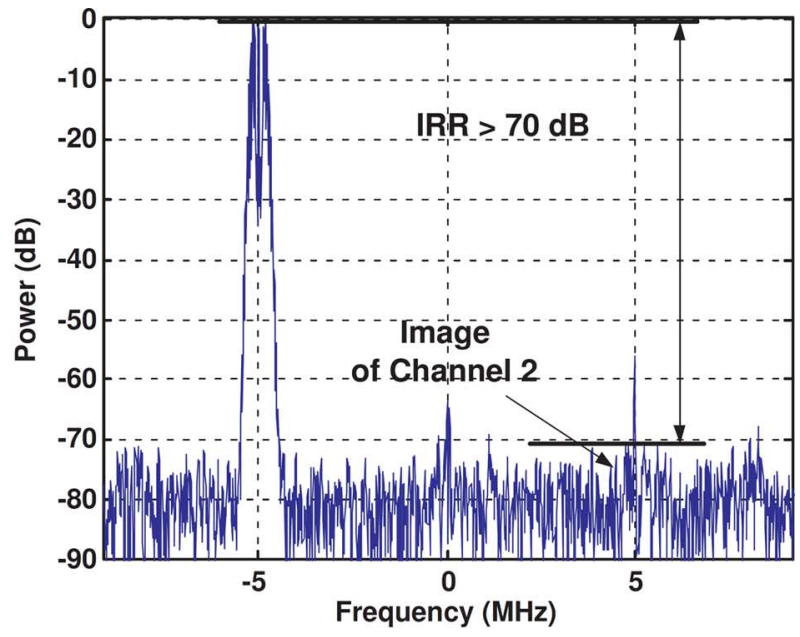

(b)

Fig. 27. Measured baseband spectrum with two carriers (a) before image rejection, and (b) after image rejection.

\section{E. Differential Noise Analysis}

Since the LNA drives the mixers and the TIAs differentially, the correlation between its output noise currents must be taken into account. Simulations reveal that, if both inputs of each TIA are driven by the LNA, then the overall noise figure falls by $0.25 \mathrm{~dB}$. This is because $I_{n 2}$ in Fig. 14 appears with the same gain and opposite phase at $\mathrm{X}$ and $\mathrm{Y}$ but is mixed with the $0^{\circ}$ and $180^{\circ}$ phases of the LO, thus acting as a commonmode baseband component. To account for this effect, the term $(\gamma+1) / G_{m 1}^{2} R_{S} R_{\text {mix }}$ in (12) must be omitted.

The noise of the TIA plays a critical role in the receiver's performance. From (12), the TIA core amplifier's input noise voltage must be limited to about $1.92 \mathrm{nV} / \sqrt{\mathrm{Hz}}$ if an overall $\mathrm{NF}$ of $3 \mathrm{~dB}$ is targeted. An important advantage of the complementary TIA implementation shown in Fig. 12 over one with constant current-source loads is that it exhibits less input-referred noise for a given bias current. A tail current of $2 \mathrm{~mA}$ allows $V_{n, \text { in,comp }}$ to be less than $1.63 \mathrm{nV} / \sqrt{\mathrm{Hz}}$.

\section{F. Noise Figure Comparison}

The proposed broadband LNA is capable of achieving a noise figure less than $1+\gamma$. To appreciate this point, we compare the circuit with the resistive-feedback and noise-cancelling topologies shown in Fig. 18.

In Fig. 18(a), the NF is approximately equal to $1+\gamma$ $+4 R_{S} / R_{F}$, exceeding $3 \mathrm{~dB}$. In Fig. 18(b), one can choose a higher transconductance for $M_{3}$ than for $M_{1}$ and a proportionally smaller $R_{2}$ than $R_{1}$, achieving [25] an NF equal to $1+\gamma R_{2} / R_{1}+R_{S} / R_{1}+R_{S} R_{2} / R_{1}^{2}+\gamma g_{m 2} R_{S}$. Unfortunately, the unequal values of $R_{1}$ and $R_{2}$ prohibit cancellation of the noise of $M_{1}$ if the circuit is followed by a balanced passive mixer and baseband TIA. If we consider only the LNA noise at $f_{\mathrm{LO}}$ for simplicity, the NF rises to $1+\gamma$ $+R_{S} / R_{1}+R_{S} R_{2} / R_{1}^{2}+\gamma g_{m 2} R_{S}$.

The proposed LNA, on the other hand, exhibits a noise figure of $1+\gamma /\left(G_{m 1} R_{S}\right)+\gamma /\left(G_{m 1} R_{\text {mix }}\right)$. With $R_{\text {mix }} \approx R_{S}=$ $50 \Omega$ and bias currents of $4 \mathrm{~mA}, 1.5 \mathrm{~mA}$, and $0.3 \mathrm{~mA}$ in $\operatorname{Inv}_{1}$,

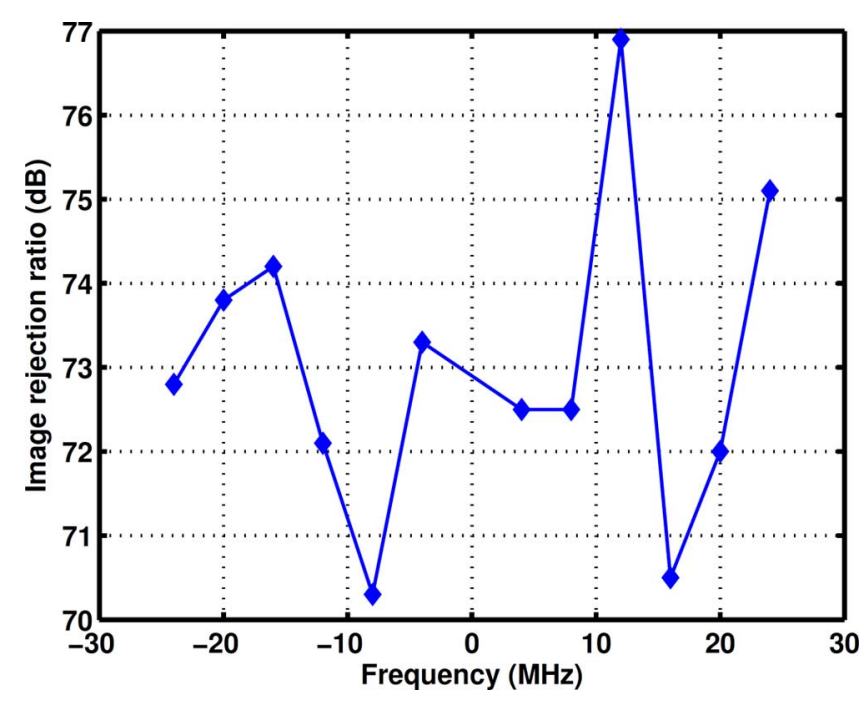

Fig. 28. Measured image-rejection ratio across baseband.

$\mathrm{Inv}_{2}$, and $\mathrm{Inv}_{3}$, respectively, we have $G_{m 1}=80 \mathrm{mS}$ and $N F$ $=1.76 \mathrm{~dB}$.

\section{FPGA IMPLEMENTATION}

From the scenario depicted in Fig. 2, we note that a narrowband blocker can appear as part of the image in the first downconversion. With a blocker power substantially higher than the desired signal power, care must be taken to minimize spectral leakage in FFT and IFFT operations of Fig. 8. In order to examine this phenomenon, we look more closely at the functions performed on the signals. As shown in Fig. 19(a), each ADC output is first multiplied by a window function, $W(t)$, then applied to an FFT engine and finally multiplied by a correction factor, $\alpha(\omega)$, in the frequency domain. In order to obtain the time-domain data, the inverse FFT of each corrected FFT frame must be taken and divided by the window function. The resulting real and imaginary parts represent the balanced I and Q data streams, respectively. For example, in our work we employ a 64-point FFT and hence choose a Blackman 


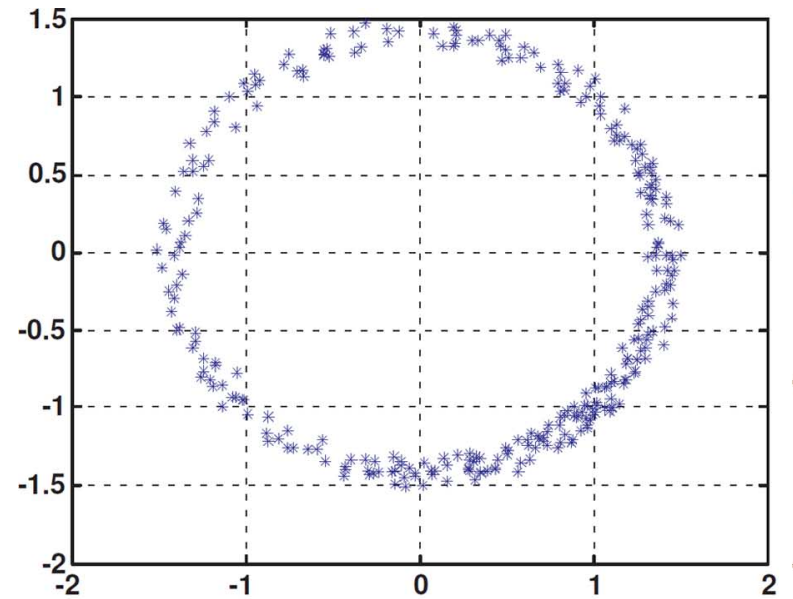

(a)

Fig. 29. Measured QPSK constellation (a) before, and (b) after calibration.

window to avoid aliasing. Since the windowed I signal is multiplied by $\alpha$ in the frequency domain, we equivalently write in the time domain $\hat{I}(t)=[I(t) W(t)] * \alpha(t)$, where $\alpha(t)$ denotes the inverse Fourier transform of $\alpha(\omega)$. For proper signal constellation formation, the windowing must eventually be undone, but the convolution with $\alpha(t)$ prohibits simple division because $\hat{I}(t) / W(t) \neq I(t) * \alpha(t)$. In fact, $\hat{I}(t) / W(t)$ suffers from enormous spectral leakage.

To resolve this issue, the I and Q processing and combining are carried out as depicted in Fig. 19(b). Here, in addition to the main I and Q paths, which generate $\alpha I(\omega)-j Q(\omega)$, a "shifting" path delays the data by half an FFT block $\left(T_{B} / 2=32\right.$ points) before windowing, FFT, and combining operations, producing $\alpha I_{d}(\omega)-j Q_{d}(\omega)$. The two composite signals are subsequently applied to IFFTs, the top path signal is delayed by $T_{B} / 2$ to match the delay of the bottom path, and the results are summed before the final inverse window.

The complexity and power consumption of the digital processing described above are of interest. The complexity is estimated conservatively from the FPGA implementation. A 64-point FFT consists of 6 stages of radix-2 butterfly structures [26]. The FFT and IFFT blocks thus require a total gate count of 84,000 , which for a gate density of $400 \mathrm{k} / \mathrm{mm}^{2}$ in $45 \mathrm{~nm}$ technology, translates to an area of roughly $0.21 \mathrm{~mm}^{2}$.

The operations required in the $\alpha$ estimator may appear rather complex. Fortunately, however, they can all be performed by bit-serial arithmetic using only adders and registers [27] because the computation of $\alpha$ can be as slow as temperature- and supplyinduced drifts in $\epsilon$ and $\theta$. Fig. 20 depicts examples for division, multiplication, and square root functions. (The word length is 20 in the implementation.) The $\alpha$ estimator consumes 3,600 gates and hence $0.01 \mathrm{~mm}^{2}$. The digital downconverter and NCO are shown in Fig. 21, requiring a total of 2,700 gates and $64 \mathrm{k}$ of memory, i.e., an area of about $0.023 \mathrm{~mm}^{2}$.

The power consumption of the digital back end arises from (a) the full-rate multipliers necessary for windowing, (b) the full-rate multipliers and accumulators used in the computation

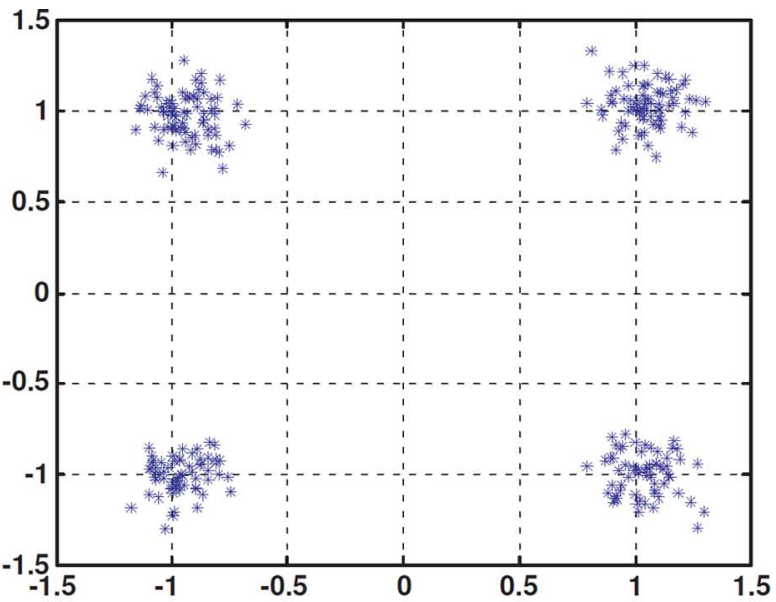

(b) of $|I|^{2},|Q|^{2}$, and $I \cdot Q$, (c) the downconverters and NCOs, and (d) the FFT and IFFT blocks. At a clock frequency of $70 \mathrm{MHz}$ in $45 \mathrm{~nm}$ technology, the first three add up to $1 \mathrm{~mW}$. From the values reported in [28] for FFT blocks in silicon, the fourth component is estimated to be $8 \mathrm{~mW}$.

\section{EXPERIMENTAL RESULTS}

This section presents the measured results for the experimental receiver system shown in Fig. 9. The CMOS prototype has been characterized both by itself and along with the receiver chain.

The CMOS prototype has been fabricated in TSMC's $45 \mathrm{~nm}$ CMOS technology and characterized with a $1 \mathrm{~V}$ supply. Shown in Fig. 22 is the die, whose core area measures $450 \mu \mathrm{m} \times 350 \mu \mathrm{m}$. Tested around $2 \mathrm{GHz}$ on a printed-circuit board, the receiver draws $15 \mathrm{~mW}: 7 \mathrm{~mW}$ in the LNA, $5 \mathrm{~mW}$ in the TIAs, and $3 \mathrm{~mW}$ in the $25 \%$-duty-cycle LO generation circuit.

Fig. 23 plots the measured noise figure for a baseband frequency from 0 to $35 \mathrm{MHz}$ and an $\mathrm{LO}$ frequency of $2 \mathrm{GHz}$. The NF ranges from $3.6 \mathrm{~dB}$ to $3.8 \mathrm{~dB}$. Fig. 24 shows the measured $S_{11}$ for $f_{\mathrm{LO}}=2 \mathrm{GHz}$, displaying a return loss of better than $-11 \mathrm{~dB}$ for $\pm 35 \mathrm{MHz}$ around $f_{\mathrm{LO}}$.

The measured $\mathrm{IIP}_{2}$ and $\mathrm{IIP}_{3}$ are plotted in Fig. 25 for two tones that are $3 \mathrm{MHz}$ apart as a function of one-tone frequency. The worst case values are $+30 \mathrm{dBm}$ and $-13 \mathrm{dBm}$ for $\mathrm{IIP}_{2}$ and $\mathrm{IIP}_{3}$, respectively. ${ }^{8}$

The complete receiver of Fig. 9 has been characterized with various types of RF inputs to evaluate the proposed image rejection algorithm. Fig. 26 shows an example with two RF channels, one containing a weak tone and another, a strong modulated signal. Fig. 27(a) depicts the downconverted (complex) spectrum before image rejection calibration, indicating an IRR of about $30 \mathrm{~dB}$. Fig. 27(b) repeats the result after calibration, showing that the image of Channel 2 is buried under

\footnotetext{
${ }^{8}$ The low value of the duplex IIP2 is attributed to imbalances between $V_{X}$ and $V_{Y}$ in Fig. 11(a).
} 


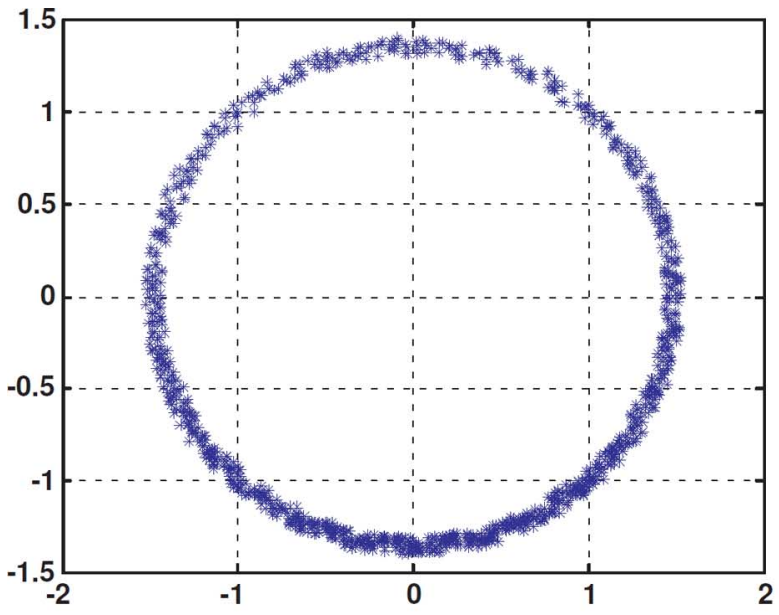

(a)

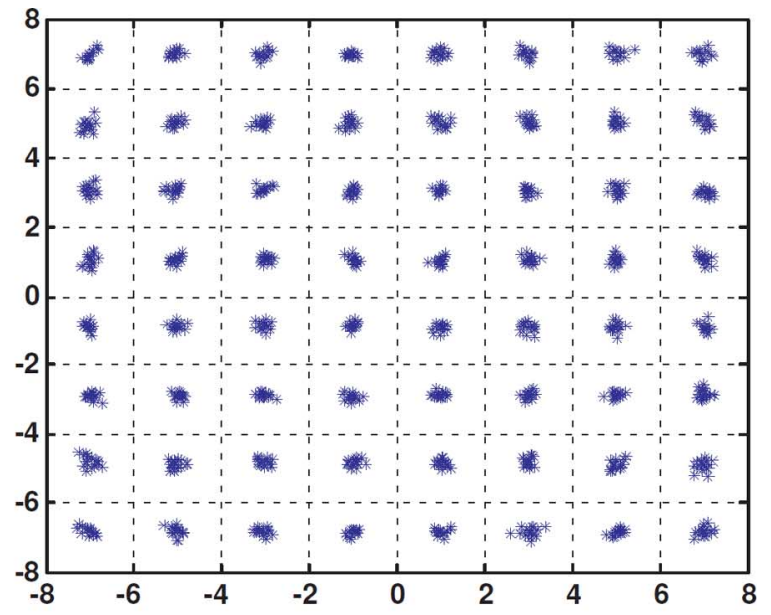

(b)

Fig. 30. Measured 64-QAM constellation (a) before, and (b) after calibration.

TABLE I

PERFormANCE SUMmARY

\begin{tabular}{|c|c|c|c|c|}
\hline & This work & $\begin{array}{c}\text { Sundstrom [4] } \\
\text { ISSCC2013 }\end{array}$ & $\begin{array}{c}\text { Din [29] } \\
\text { TCASII2013 }\end{array}$ & $\begin{array}{c}\text { Xie [30] } \\
\text { TMIT 2012 }\end{array}$ \\
\hline Standard & LTE & LTE & LTE/GSM & LTE/WCDMA \\
\hline Architecture & Block Down. & Double Conv. & Direct Conv. & Direct Conv. \\
\hline $\begin{array}{c}\text { No. of Intra- } \\
\text { Band Carriers }\end{array}$ & $>2$ & 2 & 1 & 1 \\
\hline Tech. / Supply & $45 \mathrm{~nm} / 1 \mathrm{~V}$ & $65 \mathrm{~nm} / 1.45 \mathrm{~V}$ & $65 \mathrm{~nm} / \mathrm{NA}$ & $90 \mathrm{~nm} / \mathrm{NA}$ \\
\hline Power & $15 \mathrm{~mW}$ & $68 \mathrm{~mW}$ & $80 \mathrm{~mW}^{(3)}$ & $\mathrm{NA}$ \\
\hline IIP2 (duplex) & $>40 \mathrm{dBm}$ & $>58 \mathrm{dBm}$ & $57.8 \mathrm{dBm}^{(4)}$ & $>60 \mathrm{dBm}$ \\
\hline IIP3 (half-duplex) & $2.75 \mathrm{dBm}$ & $2.4 \mathrm{dBm}$ & $-6.9 \mathrm{dBm}^{(4)}$ & $2 \mathrm{dBm}$ \\
\hline IIP2 (in-band) & $31 \mathrm{dBm}$ & $27 \mathrm{dBm}$ & $44 \mathrm{dBm}^{(4)}$ & $\mathrm{NA}$ \\
\hline IIP3 (in-band) & $-13.7 \mathrm{dBm}$ & $-15 \mathrm{dBm}$ & $-18.2 \mathrm{dBm}^{(4)}$ & $-8 \mathrm{dBm}$ \\
\hline DSB NF & $3.9 \mathrm{~dB}$ & $4.5 \mathrm{~dB}$ & $3.9 \mathrm{~dB}^{(4)}$ & $3 \mathrm{~dB}$ \\
\hline Voltage Gain & $37 \mathrm{~dB}$ & $45 \mathrm{~dB}$ & $38 \mathrm{~dB}^{(4)}$ & $60 \mathrm{~dB}$ \\
\hline IRR & $>70 \mathrm{~dB}$ & $>55 \mathrm{~dB}$ & $\mathbf{N A}$ & $\mathrm{NA}$ \\
\hline
\end{tabular}

(1) LNA + Mixers + TIAs + LO gen.

(2) Estimated from ISSCC Visual Supplement for LNA + RF mixers + IF mixers, excluding the power of all IF and baseband filters.

(3) LNA + Mixers + TIAs + LO gen.

(4) Measured at $1800 \mathrm{MHz}$.

the noise floor. (In both cases, the output spectrum is computed by the FPGA.) Fig. 28 extends these results for an IF range of $-25 \mathrm{MHz}$ to $+25 \mathrm{MHz}$.

The performance of the receiver chain has also been assessed by examining signal constellations. A QPSK signal in Channel 1 and $40 \mathrm{~dB}$ below another modulated signal in Channel 2 yields the constellations shown in Fig. 29(a) and (b) before and after calibration, respectively. Fig. 30 repeats the experiment with a 64-QAM signal, yielding an error vector magnitude of $-30.1 \mathrm{~dB}$ after calibration for a Channel 1 input power of $-76 \mathrm{dBm}$.

Table I summarizes the performance of our receiver and compares it with that of the prior art. ${ }^{9}$

\footnotetext{
${ }^{9}$ The comparison with [4] considers only relevant blocks, but, of course, the work in [4] is an integrated receive chain while our prototype consists of an integrated front-end and off-the-shelf components. The IRR in [4] is greater than $60 \mathrm{~dB}$ as averaged over one channel after calibration.
}

\section{CONCLUSION}

This paper proposes a receiver architecture that can accommodate intra-band channel aggregation for the LTE standard. The use of a new image rejection algorithm in the digital back end greatly simplifies the RF and analog sections of the design and allows calibration of frequency-dependent mismatches. A new broadband LNA topology is also presented that achieves a noise figure less than $1+\gamma$. With improvements in the performance of ADCs, it is expected that the proposed architecture can also be applied to concurrent reception in WiFi applications.

\section{REFERENCES}

[1] 3GPP, 3rd-Generation Partnership Project; Technical Specification Group Radio Access Network; Further Advancements for E-UTRA; Physical Layer Aspects (Release 9), in TS 36.814 V0.4.1, 2009. 
[2] 3GPP, 3rd-Generation Partnership Project; Technical Specification Group Radio Access Network; LTE; Evolved Universal Terrestrial Radio Access (E-UTRA); User Equipment (UE) radio transmission and reception (Release 11), 3GPP TS 36.101 V11.5.0, Jul. 2013.

[3] F. Adachi et al., "Frequency-domain equalization for broadband single-carrier multiple access," IEICE Tran. Commun., vol. E92-B, no. 5, pp. 1441-1456, May 2009.

[4] L. Sundstrom et al., "A receiver for LTE rel-11 and beyond supporting non-contiguous carrier aggregation," in IEEE ISSCC Dig. Tech. Papers, Feb. 2013, pp. 336-337.

[5] L. Sundstrom et al., "Complex IF harmonic rejection mixer for noncontiguous dual carrier reception in $65 \mathrm{~nm}$ CMOS," IEEE J. Solid-State Circuits, vol. 48, no. 7, pp. 1659-1668, Jul. 2013.

[6] M. Anderson et al., "A 4.75-34.75 MHz digitally tunable active-RC LPF for $>60 \mathrm{~dB}$ mean RX IRR in $65 \mathrm{~nm}$ CMOS," in Proc. ESSCIRC, Sep. 2012, pp. 470-473.

[7] L. R. Wilhelmsson et al., "Design of a configurable complex IF receiver supporting LTE carrier aggregation," in Proc. IEEE Veh. Technol. Conf., Jun. 2013, pp. 1-5.

[8] 3GPP, 3rd-Generation Partnership Project; Technical Specification Group Radio Access Network; LTE; Evolved Universal Terrestrial Radio Access (E-UTRA); Base Station (BS) radio transmission and reception (Release 9), 3GPP TS 36.104 V9.4.0, Jul. 2010.

[9] R. Kapusta et al., "A 14b 80 MS/s SAR ADC with 73.6 dB SNDR," IEEE J. Solid-State Circuits, vol. 48, no. 12, pp. 3059-3066, Dec. 2013.

[10] G. Taylor et al., "A reconfigurable mostly-digital delta-sigma ADC with a worst-case FOM of $160 \mathrm{~dB}$," IEEE J. Solid-State Circuits, vol. 48, no. 4, pp. 983-995, Apr. 2013.

[11] M. Elmala and S. Embabi, "Calibration of phase and gain mismatches in Weaver image-reject receiver," IEEE J. Solid-State Circuits, vol. 39, no. 2, pp. 283-289, Feb. 2004.

[12] S. Lerstaveesin and B. Song, "A complex image rejection circuit with sign detection only," IEEE J. Solid-State Circuits, vol. 41, no. 12, pp. 2693-2702, Dec. 2006.

[13] C. Heng et al., "A CMOS TV tuner/demodulator IC with digital image rejection," IEEE J. Solid-State Circuits, vol. 40, no. 12, pp. 2525-2535, Dec. 2005.

[14] I. Elahi et al., "I/Q mismatch compensation using adaptive decorrelation in a low-IF receiver in $90 \mathrm{~nm}$ CMOS process," IEEE J. Solid-State Circuits, vol. 41, no. 2, pp. 395-404, Feb. 2006.

[15] L. Yu and W. M. Snelgrove, "A novel adaptive mismatch cancellation system for quadrature IF radio receivers," IEEE Trans. Circuits Syst. II, vol. 46, no. 6, pp. 789-801, Jun. 1999.

[16] S. V. Gervan and D. V. Compernolle, "Signal separation by symmetric adaptive decorrelation: Stability, convergence, and uniqueness," IEEE Trans. Signal Process., vol. 43, no. 7, pp. 1602-1612, Jul. 1995.

[17] Texas Instruments, ADS62P549, Dual-Channel 14-12-bit 250-210MSPS ADC with DDR LVDS and parallel CMOS outputs.

[18] A. N. Karanicolas, "A 2.7-V $900 \mathrm{MHz}$ CMOS LNA and mixer," IEEE J. Solid-State Circuits, vol. 31, no. 12, pp. 1939-1944, Dec. 1996.

[19] D. Murphy et al., "A blocker-tolerant, noise-cancelling receiver suitable for wideband wireless applications," IEEE J. Solid-State Circuits, vol. 47, no. 12, pp. 2943-2963, Dec. 2012.

[20] B. Razavi, "Cognitive radio design challenges and techniques," IEEE J. Solid-State Circuits, vol. 45, no. 8, pp. 1542-1553, Aug. 2010.

[21] J. W. Park and B. Razavi, "A harmonic-rejecting CMOS LNA for broadband radios," IEEE J. Solid-State Circuits, vol. 48, no. 4, pp. 1072-1084, Apr. 2013.

[22] A. Mirzaei et al., "Architectural evolution of integrated M-phase high-Q bandpass filters," IEEE Trans. Circuits Syst. I, vol. 59, no. 1, pp. 52-65, Jan. 2012.

[23] D. Murphy et al., "An LTV analysis of the frequency translational noise-cancelling receiver," IEEE Trans. Circuits Syst. I, no. 7, pp. 1-14, Jul. 2013.

[24] C. Andrews and A. C. Molnar, "Implications of passive mixer transparency for impedance matching and noise figure in passive mixer-first receivers," IEEE Trans. Circuits Syst. I, vol. 57, no. 12, pp. 3092-3103, Dec. 2010.

[25] F. Bruccoleri et al., "Wide-band CMOS low-noise amplifier exploiting thermal noise cancelling," IEEE J. Solid-State Circuits, vol. 39, no. 2, pp. 275-282, Feb. 2004.

[26] A. V. Oppenheim, R. W. Schafer, and J. R. Buck, Discrete-Time Signal Processing, 2nd ed. Englewood Cliffs, NJ, USA: Prentice Hall, 1989, ch. 9 .
[27] K. Parhi, VLSI Digital Signal Processing Systems: Design and Implementation. New York, NY, USA: Wiley, 1999, ch. 13.

[28] C.-H. Yang et al., "Power and area minimization of reconfigurable FFT processors: A 3GPP-LTE example," IEEE J. Solid-State Circuits, vol. 47, no. 3, pp. 757-768, Mar. 2012.

[29] I. Din et al., "Wideband SAW-less receiver front-end with harmonic rejection mixer in $65 \mathrm{~nm}$ CMOS," IEEE Trans. Circuits Syst. II, no. 5, pp. 242-246, May 2013.

[30] H. Xie et al., "Single-chip multiband EGPRS and SAW-less LTE WCDMA CMOS receiver with diversity," IEEE Trans. Microw. Theory Tech., pp. 1390-1396, May 2012.

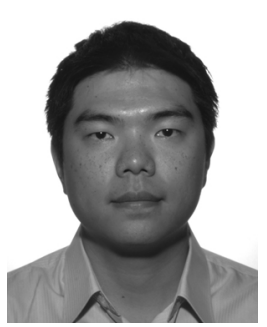

Sy-Chyuan Hwu received the B.S.E.E. and M.S.E.E. degrees from National Taiwan University, Taipei, Taiwan, in 2003 and 2005, respectively. He was with MediaTek Inc. until 2010. He received the Ph.D.E.E. degree from the University of California, Los Angeles, CA, USA, in 2013.

Since 2014, he has joined Qualcomm Technologies Inc, San Diego, CA, USA. His research interest includes analog, RF, and millimeter-wave integrated circuit design for wireless transceivers.

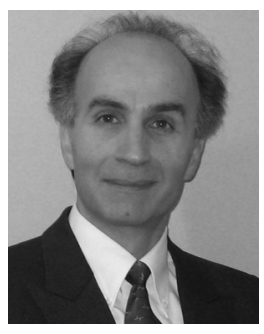

Behzad Razavi (S'87-M'90-SM'00-F'03) received the B.S.E.E. degree from Sharif University of Technology, Tehran, Iran, in 1985 and the M.S.E.E. and Ph.D.E.E. degrees from Stanford University, Stanford, CA, USA, in 1988 and 1992, respectively.

He was with AT\&T Bell Laboratories and Hewlett-Packard Laboratories until 1996. Since 1996, he has been Associate Professor and subsequently Professor of electrical engineering at University of California, Los Angeles. His current research includes wireless transceivers, frequency synthesizers, phase-locking and clock recovery for high-speed data communications, and data converters. He was an Adjunct Professor at Princeton University from 1992 to 1994, and at Stanford University in 1995.

Prof. Razavi served on the Technical Program Committees of the International Solid-State Circuits Conference (ISSCC) from 1993 to 2002 and VLSI Circuits Symposium from 1998 to 2002. He has also served as Guest Editor and Associate Editor of the IEEE Journal of Solid-State Circuits, IEEE TRANSACTIONS ON CIRCUITS AND SYSTEMS, and the International Journal of High Speed Electronics. He received the Beatrice Winner Award for Editorial Excellence at the 1994 ISSCC, the best paper award at the 1994 European Solid-State Circuits Conference, the best panel award at the 1995 and 1997 ISSCC, the TRW Innovative Teaching Award in 1997, the best paper award at the IEEE Custom Integrated Circuits Conference in 1998, and the McGraw-Hill First Edition of the Year Award in 2001. He was the co-recipient of both the Jack Kilby Outstanding Student Paper Award and the Beatrice Winner Award for Editorial Excellence at the 2001 ISSCC. He received the Lockheed Martin Excellence in Teaching Award in 2006, the UCLA Faculty Senate Teaching Award in 2007, and the CICC Best Invited Paper Award in 2009 and in 2012. He was the co-recipient of the 2012 VLSI Circuits Symposium Best Student Paper Award and the 2013 CICC Best Paper Award. He was also recognized as one of the top 10 authors in the 50-year history of ISSCC. He received the 2012 Donald Pederson Award in Solid-State Circuits. He was also the recipient of the American Society for Engineering Education PSW Teaching Award in 2014

Prof. Razavi has served as an IEEE Distinguished Lecturer and is a Fellow of IEEE. He is the author of Principles of Data Conversion System Design (IEEE Press, 1995), RF Microelectronics (Prentice Hall, 1998, 2012) (translated to Chinese, Japanese, and Korean), Design of Analog CMOS Integrated Circuits (McGraw-Hill, 2001) (translated to Chinese, Japanese, and Korean), Design of Integrated Circuits for Optical Communications (McGraw-Hill, 2003, Wiley, 2012), and Fundamentals of Microelectronics (Wiley, 2006) (translated to Korean and Portuguese), and the editor of Monolithic Phase-Locked Loops and Clock Recovery Circuits (IEEE Press, 1996), and Phase-Locking in High-Performance Systems (IEEE Press, 2003). 